\title{
Development of the kinetic molecular theory of gases concept inventory: Preliminary results on university students' misconceptions
}

\author{
Nataša Erceg, ${ }^{1, *}$ Ivica Aviani, ${ }^{2}$ Vanes Mešić, ${ }^{3}$ Matko Glunčić, ${ }^{4}$ and Gordana Žauhar ${ }^{5}$ \\ ${ }^{1}$ Department of Physics, University of Rijeka, R. Matejčić 2, 51000 Rijeka, Croatia \\ ${ }^{2}$ Institute of Physics, Bijenička c. 46, Hr-10002 Zagreb, Croatia \\ and Faculty of Science, University of Split, R. Boškovića 33, 21000 Split, Croatia \\ ${ }^{3}$ Faculty of Science, University of Sarajevo, Zmaja od Bosne 33-35, \\ 71000 Sarajevo, Bosnia and Herzegovina \\ ${ }^{4}$ Department of Physics, Faculty of Science, University of Zagreb, \\ Bijenička 32, HR-10000 Zagreb, Croatia \\ ${ }^{5}$ Department of Physics, University of Rijeka, R. Matejčic 2, 51000 Rijeka, Croatia \\ and Faculty of Medicine, University of Rijeka, Braće Branchetta 20, 51000 Rijeka, Croatia
}

(Received 28 June 2016; published 16 November 2016)

\begin{abstract}
In this study, we investigated students' understanding of concepts related to the microscopic model of gas. We thoroughly reviewed the relevant literature and conducted think alouds with students by asking them to answer open-ended questions about the kinetic molecular theory of gases. Thereafter, we transformed the open-ended questions into multiple-choice questions, whereby distractors were based on the results of the think alouds. Thus, we obtained a set of 22 questions, which constitutes our current version of the kinetic molecular theory of gases concept inventory. The inventory has been administered to 250 students from different universities in Croatia, and its content validity has been investigated trough physics teacher surveys. The results of our study not only corroborate the existence of some already known student misconceptions, but also reveal new insights about a great spectrum of students' misconceptions that had not been reported in earlier research (e.g., misconceptions about intermolecular potential energy and molecular velocity distribution). Moreover, we identified similar distribution of students' responses across the surveyed student groups, despite the fact that they had been enrolled in different curricular environments.
\end{abstract}

DOI: 10.1103/PhysRevPhysEducRes.12.020139

\section{INTRODUCTION}

Earlier research on students' understanding of basic thermal concepts showed that students exhibit numerous difficulties [1], which are reflected in making no distinctions between basic concepts, in ideas that conflict with accepted scientific ideas [2], or in application of irrelevant theories [3-5]. For example, it is common that students do not differentiate between the concepts of temperature and heat [2-12], often conceptualizing temperature as the amount of heat contained inside a body or something that flows from one body to another [11]. Furthermore, students often mistakenly consider the concept of heat to be the same as the concept of thermal energy, imagining heat as a form of energy that is determined by temperature, instead of considering it as energy that passes from one body to another due to temperature difference $[4,13,14]$. Because

\footnotetext{
*nerceg@phy.uniri.hr
}

Published by the American Physical Society under the terms of the Creative Commons Attribution 4.0 International license. Further distribution of this work must maintain attribution to the author(s) and the published article's title, journal citation, and DOI. students do not distinguish between thermal internal energy and heat, they often mistakenly believe that the thermal insulation of the container with gas will prevent a change of the gas thermal internal energy, or gas temperature, even if the volume of the gas is changed [4]. This further creates a lack of understanding of the concept of thermal insulation in the sense of preventing heat exchange between the system and the environment, and the difficulty of applying the concept of work associated with the change of gas thermal internal energy or gas temperature in the adiabatic process [4]. Some students argue that the system energy always remains constant [15]. In general, many students do not understand that heat and work have independent meaning with regard to the transfer of energy, and that the change in thermal internal energy depends on them. This creates difficulties in understanding and application of the first law of thermodynamics [2-4]. Therefore, it often happens that students interpret a process in a way that instead of the first law of thermodynamics, they mistakenly use other laws, for example, the ideal gas law [3-5]. Students do not use it only in irrelevant situations for gases, but also for solids or liquids [16]. When it comes to the concept of entropy, it should be pointed out that there is still a lot of disagreement amongst experts on the mere 
definition of this more than 100 year old concept [17]. For example, there are conflicting opinions on whether or not entropy of a classical system should be defined in terms of the logarithm of phase space volume. Although according to Neumann (as cited in Ref. [18]) "Nobody really knows what entropy really is," Swendsen [17] suggests that entropy should be defined in terms of the logarithm of the probability of macrostates of composite systems. This approach to defining entropy is in line with the definition of entropy provided by Boltzmann as far back as 1877 . In view of the above, it is not surprising that students have difficulties to develop an understanding of entropy [19-22]. For example, students erroneously associate entropy with the order and disorder of a system [20,22] and believe that entropy is a conserved quantity [21]. Moreover, they do not use a single simple model of entropy, but rather use a variety of conceptual resources and shift between resources, which leads them to contradictory predictions [20].

Sources of students' difficulties with thermodynamics have been thoroughly discussed in earlier studies. They are often associated with incorrect language in nonphysical textbooks, in standard documents, and in everyday speech $[23,24]$, where, for example, heat and temperature are used as synonyms. Additionally, in some textbooks [16] the concept of thermal energy is not differentiated from the concept of heat or from the concept of internal energy.

One additional source for many of the students' difficulties with macrolevel concepts of thermodynamics could be related to their deficient microlevel models of a substance [2]. Students begin to develop ideas about the particulate nature of matter already in primary school. Most of them become aware of the particulate nature of matter before entering university, where they continue to develop their understanding of kinetic molecular theory within introductory physics and chemistry courses, as well as within specialized courses, such as thermodynamics and statistical physics [25]. However, it has been shown that even after being exposed to instruction about kinetic molecular concepts for many years, students often exhibit difficulties in comprehending and applying the most important features of micro-models [2]. Many of the students' misconceptions about kinetic molecular theory are described in earlier studies. For example, it has been shown that students often incorrectly think that colliding of particles produces heat or kinetic energy $[3,13,25]$. Such a conception of basic microscopic processes can give rise to further misconceptions, not only related to the average kinetic energy per molecule, but also to the concept of gas temperature $[4,5,13,25,26]$. Considering that the temperature increases due to more frequent collisions among the particles within the reduced volume, students incorrectly assume that the thermal internal energy change is achieved through the interaction within the system, rather than through the interaction between the system and its environment [4,25]. Robertson and Shaffer [27] found that students' misconceptions on motion of gas particles often cause them to incorrectly predict how the volume of a gas changes as a result of temperature change. Furthermore, results of survey research [25] indicate that many students do not have an adequate qualitative model of gas pressure.

According to Meltzer [3] and Loverude, Kautz, and Heron [4], a poor understanding of interrelated thermodynamics concepts and their interpretations from macroscopic and microscopic perspectives, makes it difficult for many students in introductory physics courses to solve problems situated in subjectively new contexts. Therefore, it is suggested that our knowledge about students' misconceptions and difficulties should serve as a starting point when planning for effective teaching of physics $[5,9,25,28,29]$. In other words, it is necessary to systematically explore students' conceptual framework related to kinetic molecular theory, and then to organize conceptualization or conceptual change in line with the obtained model of student functioning [29]. In this regard, it should be noted that there are already many concept inventories in the area of thermodynamics. They are mainly aimed for assessing students' understanding of macroscopic phenomena related to heating of substances and heat transfer, containing items which can be solved without using the real gas model (Table I).

TABLE I. The names and descriptions of concept inventories in the area of thermodynamics.

\begin{tabular}{lc}
\hline \hline Name & Description \\
\hline Thermodynamics Concept Inventory (TCI) [30] & $\begin{array}{c}\text { It assesses undergraduate engineering student understanding of } \\
\text { fundamental thermodynamics concepts. }\end{array}$ \\
Introductory Thermal Concept Evaluation (ITCE) [31] & $\begin{array}{c}\text { It assesses 15-18 year old student understanding of thermodynamics } \\
\text { concepts. } \\
\text { Heat and Temperature Concept Evaluation (HTCE) [32] }\end{array}$ \\
$\begin{array}{l}\text { It assesses intro college level student understanding on concepts of heat, } \\
\text { temperature, and heat flow. } \\
\text { It assesses undergraduate engineering student understanding on concepts } \\
\text { of heat transfer. }\end{array}$ \\
Thermal and Transport Concept Inventory (TTCI) [34] & $\begin{array}{c}\text { It assesses undergraduate engineering student understanding on concepts } \\
\text { of heat transfer, fluid mechanics and thermodynamics. } \\
\text { It assesses undergraduate engineering student understanding on concepts } \\
\text { of temperature, heat and energy. }\end{array}$ \\
\hline \hline
\end{tabular}


However, despite the fact that misconceptions about kinetic molecular theory are relatively well documented, there are, according to our knowledge, no conceptual inventories that could be easily used for identifying students' conceptual frameworks about kinetic molecular theory when teaching introductory physics to large groups of university students.

The motivation for our research arose from informal discussions with students and physics teachers, who had difficulties understanding a primary school textbook problem [35], from the perspective of the kinetic molecular theory of a substance. Concretely, in the quoted textbook problem, students are asked to determine the temperature and compare thermal internal energies of the same amounts of liquid water, water vapor, and ice which are the result of melting ice in a glass of water at room temperature. Although students and teachers declaratively know that the temperature of the ice and water mixture is $0^{\circ} \mathrm{C}$ and that it does not change as the ice melts, throughout our discussion many of them did not use that knowledge. Quite opposite, almost all of them provided the incorrect answer that ice has the lowest temperature, followed by liquid water, and water vapor. On the other hand, they correctly estimated that the thermal internal energy of an amount of ice is the lowest, and the thermal internal energy of the same amount of vapor is the greatest, for the given situation. However, at the same time, our discussion partners completely ignored the intermolecular potential energy, which considerably contributes to thermal internal energy in the given situation. Specifically, they mistakenly believed that liquid water and vapor had a higher thermal internal energy than ice only due to higher kinetic energy of their molecules, which is in line with their wrong answer to the temperature-related part of the quoted question.

In our opinion, our discussion partners' difficulties in providing a correct answer to the ice-water mixture problem could stem from the fact that the concept of thermal internal energy is typically addressed within the model of ideal gases, in which intermolecular potential energy is neglected. Thus, one possible explanation for students' difficulties could be that they reasoned about that ice-water mixture problem from a perspective of ideal gases. Consequently, we decided to conduct an investigation with the aim of gaining a more detailed insight into the students' understanding of real gases that goes beyond understanding of the ideal gas model.

In this study we investigated students' understanding of concepts related to the simplest real model, i.e., we examined their understanding of the kinetic molecular theory of gases (KMTG). Taking into account that there is, according to our knowledge, no earlier research on students' understanding of the concept of intermolecular potential energy, we decided to devote most attention to exploring students' understanding of that concept.

\section{METHODS}

The most important aspects of the methodology used in our study are represented in Table II. In the sections that follow, we attempt to provide additional important information about our methodological approach to this study.

\section{A. Participants}

Based on our teaching experience and review of relevant literature, we first developed an open-ended version of the

TABLE II. Most important phases of KMTG inventory development.

1. Defining the construct which is to be measured

The construct which we attempted to measure by means of KMTG inventory is the understanding of the kinetic molecular theory of gases. In order to delimit the content domain and implicitly set the general assessment objectives, we reviewed the relevant PER literature on teaching and learning thermodynamics, and we also reviewed the diverse introductory physics curricula, at the university level.

2. Identifying the behavior which corresponds to different levels of the defined construct

Based on the above mentioned analyses, we attempted to design open-ended tasks that could be used for assessing the students' location on the earlier defined latent trait (understanding of the kinetic molecular theory of gases).

3. Trying out the open-ended version of the inventory; Identifying behavior outcomes

We conducted individual one-on-one think-alouds with university students $(N=8)$, with the aim of probing students' reactions to the open-ended version of the inventory. Students answers were categorized, and prototypical answers were chosen to represent distracters.

4. Evaluating the close-ended version of the inventory

Four groups of university students $(N=250)$, representing four different curricula, have been surveyed with the aim of evaluating the close-ended version of the KMTG inventory. Results of the survey were used for identifying psychometrical characteristics of the developed inventory, as well as for gaining feedback on its possible improvement. Analysis of student answers provided us with information about some prominent student misconceptions in the field of gases.

5. Expert survey

We asked 18 physics teachers and professors to evaluate the close-ended version of the KMTG inventory. Their answers were used for estimating content validity of our instrument and they will be used for purposes of assessing the teacher buy-in of the inventory in future versions. 
KMTG concept inventory. That concept inventory has been used for conducting think alouds with 8 students (volunteers) from different years of undergraduate and graduate physics studies at University of Rijeka (Croatia). Each of these students had learned about kinetic molecular theory in at least one of the university courses. Common to all these interviewees was that they had been earlier enrolled in the Physics IV course (Heat and Introduction to Statistical Physics), which consists of $60 \mathrm{~h}$ of lecture and $30 \mathrm{~h}$ of recitations. The think alouds lasted from $25 \mathrm{~min}$ to $75 \mathrm{~min}$ (average duration $45 \mathrm{~min}$ ). Each think aloud was audio taped and transcribed.

The analysis of think-aloud transcripts helped us to prepare distractors for the closed-ended version of the KMTG concept inventory. The closed-ended version of the KMTG concept inventory was administered to four groups of respondents (MED, EIT, EDU, and RES; see Table III) consisting of altogether 250 university students. The nonrandom convenience sampling technique [36] was used.

The group MED (see Table III) included a total of 102 first-year undergraduate students who attended the Medical Physics and Biophysics course. This is an introductory course given during the first trimester of integrated undergraduate and graduate university study of medicine, and consists of $30 \mathrm{~h}$ of lectures, $20 \mathrm{~h}$ of seminars, and $25 \mathrm{~h}$ of laboratory exercises. During the course students acquire knowledge about the physical principles that are needed for the better understanding of processes in anatomy, biochemistry, physiology, histology, pathology, and other areas. The students acquire the ability to explain how the body works as a thermodynamic system, to apply the energy conservation law in the calculation of energy equilibrium of a body, to explain the physical phenomena on which the cellular transport mechanisms are based, and to understand how we walk, talk, see, and hear. Thermodynamics and the kinetic molecular theory of gases makes only a small part of the overall course content, and from a total of 30 class hours of lectures only $2 \mathrm{~h}$ include the contents of thermodynamics and $1 \mathrm{~h}$ is devoted to kinetic molecular theory.

The group EIT (Table III) included a total of 64 thirdyear undergraduate students, who attended the General Physics 1 course. This is an introductory course performed during the first year (2nd semester) of undergraduate study of Electrical Engineering and Information Technology. Students attend $45 \mathrm{~h}$ of lectures, $30 \mathrm{~h}$ of auditory exercises and $15 \mathrm{~h}$ of seminars. From total of 45 class hours of lectures, $10 \mathrm{~h}$ include the contents of thermodynamics and $2 \mathrm{~h}$ are devoted to kinetic molecular theory. Throughout the course, students acquire the knowledge of the basic physical laws and concepts from the field of classical mechanics, fluid mechanics, and thermodynamics. By the end of the course, students are expected to have the capacity to define basic physical quantities, laws, and concepts in thermodynamics; apply basic laws of thermodynamics to solve numerical tasks for known systems in thermodynamics; set mathematical formulations of basic physical models in thermodynamics; and measure basic thermodynamical quantities.

The group EDU (Table III) included a total of 64 thirdyear undergraduate students, who attended the General Physics 4 course. This is an introductory course given during the second year (4th semester) of integral undergraduate and graduate study of physics-Educational studies. Students attend $60 \mathrm{~h}$ of lectures, $30 \mathrm{~h}$ of auditory exercises, and $15 \mathrm{~h}$ of seminars. During the course students

TABLE III. Description of subgroups' curricula related to thermodynamics. For each group we provide information about: the number of hours of lectures (L), auditory exercises (AE), seminars (S) or laboratory exercises (LE), number of class hours devoted to thermodynamics, number of class hours devoted to kinetic molecular theory.

\begin{tabular}{|c|c|c|c|c|}
\hline $\begin{array}{l}\text { Name of the } \\
\text { group and } \\
\text { number of } \\
\text { participants }\end{array}$ & $\begin{array}{l}\text { Name and year of the } \\
\text { university study programme }\end{array}$ & $\begin{array}{c}\text { Name of the course, the number } \\
\text { of hours ( } \mathrm{L}, \mathrm{AE}, \mathrm{S}, \mathrm{LE}) \text { and } \\
\text { place in curriculum }{ }^{\text {a }}\end{array}$ & $\begin{array}{l}\text { Number of the } \\
\text { class hours } \\
\text { devoted to } \\
\text { thermodynamics }\end{array}$ & $\begin{array}{l}\text { Number of the } \\
\text { course hours } \\
\text { related to kinetic } \\
\text { molecular theory }\end{array}$ \\
\hline $\begin{array}{l}\mathrm{MED} \\
\qquad\left(N_{\mathrm{MED}}=102\right)\end{array}$ & $\begin{array}{l}\text { Integrated undergraduate and } \\
\text { graduate university study of } \\
\text { Medicine (1st year) }\end{array}$ & $\begin{array}{l}\text { Medical Physics and Biophysics } \\
(30 \mathrm{~L}+25 \mathrm{LE}+20 \mathrm{~S}) 1 \text { st year, } \\
1 \text { st trimester }\end{array}$ & $2 \mathrm{~L}$ & $1 \mathrm{~L}$ \\
\hline $\begin{array}{l}\mathrm{EIT} \\
\qquad\left(N_{\mathrm{EIT}}=64\right)\end{array}$ & $\begin{array}{l}\text { Undergraduate study programme } \\
\text { Electrical Engineering and } \\
\text { Information Technology } \\
\text { (2nd year) }\end{array}$ & $\begin{array}{l}\text { General Physics } 1 \\
\quad(45 \mathrm{~L}+30 \mathrm{AE}+15 \mathrm{LE}) 1 \text { st year, } \\
\text { 2nd semester }\end{array}$ & $10 \mathrm{~L}$ & $2 \mathrm{~L}$ \\
\hline $\begin{array}{l}\mathrm{EDU} \\
\qquad\left(N_{\mathrm{EDU}}=64\right)\end{array}$ & $\begin{array}{l}\text { Integral undergraduate and } \\
\text { graduate study of physics- } \\
\text { Educational studies (3rd year) }\end{array}$ & $\begin{array}{l}\text { General Physics } 4 \\
\quad(60 \mathrm{~L}+30 \mathrm{AE}+15 \mathrm{~S}) \text { 2nd year, } \\
\quad \text { 4th semester }\end{array}$ & $30 \mathrm{~L}$ & $2 \mathrm{~L}$ \\
\hline $\begin{array}{l}\mathrm{RES} \\
\qquad\left(N_{\mathrm{RES}}=20\right)\end{array}$ & $\begin{array}{l}\text { Integral undergraduate and } \\
\text { graduate study of physics- } \\
\text { Research oriented (3rd year) }\end{array}$ & $\begin{array}{l}\text { General Physics } 4 \\
\quad(60 \mathrm{~L}+30 \mathrm{AE}+15 \mathrm{~S}) \text { 2nd year, } \\
\quad \text { 4th semester }\end{array}$ & $60 \mathrm{~L}$ & $4 \mathrm{~L}$ \\
\hline
\end{tabular}

\footnotetext{
${ }^{\mathrm{a}}$ course covers the contents of thermodynamics and hence the kinetic molecular theory.
} 
acquire operational knowledge related to solving problems in thermodynamics and the basics of modern physics. Thermodynamics makes up half of the course content; however, from a total of 60 class hours of lectures only $2 \mathrm{~h}$ are devoted to the kinetic molecular theory of gases. By the end of the course, students are expected to have the capacity to understand the concept of a heat engine and apply it to real-life systems, demonstrate knowledge of the description of a general thermodynamic system, understand the concept of entropy and connect it to relevant quantities in a particular system, and understand the basic composition of matter and atomic, as well as nuclear processes.

The group RES (Table III) included a total of 20 third-year undergraduate students enrolled to the integral undergraduate and graduate study of physics-Research oriented. They attended the General Physics 4 course which consists of $60 \mathrm{~h}$ of lectures, $30 \mathrm{~h}$ of auditory exercises, and $15 \mathrm{~h}$ of seminars, which are performed during the second year (4th semester). Throughout the course, the RES students acquire theoretical and experimental knowledge of the basics in statistical physics and thermodynamics. The whole course includes only $4 \mathrm{~h}$ devoted to the kinetic molecular theory of gases. By the end of the course, students are expected to have the capacity to develop a simple physical model in statistical physics and thermodynamics, to set mathematical formulation of a given physical model in statistical physics and thermodynamics, solve numerical tasks for known systems in statistical physics and thermodynamics, demonstrate operational use of statistical distributions relevant to thermodynamics, demonstrate knowledge of the properties of the classical ideal gas, demonstrate knowledge of equilibrium and irreversible processes, demonstrate knowledge of internal combustion engines, and demonstrate basic knowledge of real gases and phase transitions.

\section{B. Concept inventory design}

The current version of the closed-ended KMTG concept inventory (see Appendix A, i.e. Figs. 10-12) consists of 22 multiple choice questions designed for measuring students' conceptual understanding of the kinetic molecular theory of gases. The creation of the current version of the KMTG concept inventory was preceded by the implementation of think alouds [37]. Altogether eight physics students from the University of Rijeka agreed to volunteer in our think alouds. The one-on-one think alouds, without the presence of third persons, were conducted by the first author of this paper. Each interviewee has been given the written, openended version of the KMTG concept inventory, consisting of the same 22 questions as the current version of the inventory. For example, in task 1 (see Appendix A) students were required to answer the following question "What is the difference between an ideal gas and a gas when it comes to their structure and interactions between particles?" After reading aloud each inventory question, the students were expected to provide an oral answer to that question. During the think aloud, the interviewer was probing students as infrequently as possible. When silences continued for several seconds, the interviewer merely asked the students to keep talking. Once the think-aloud process was completed, each interviewee was typically asked for further clarification. Specifically, they were asked follow-up questions, such as "Why do you think so?", "Could you additionally explain or justify your answer?", etc. In the test sheet, below each task statement we left some blank space, where the interviewees could illustrate their ideas by drawings (e.g., drawings were required in task 2: "How would you illustrate the path of the gas molecule due to its thermal motion in a container represented by the square frame?") or use the blank space for making some preliminary notes for purposes of facilitating reasoning. In addition, all think alouds were audio taped and transcribed. Finally, the data obtained through think alouds has been analyzed. We categorized the answers for each of the tasks, whereby each category reflected answers that shared some specific (physically incorrect) idea. Categories that occurred most frequently were used as a basis for formulating distractors, i.e., for creating the closed-ended version of the KMTG concept inventory (Appendix A). Specifically, original student answers that were considered to prototypically represent the categories of student ideas were later used as distracters in the closed-ended version of our inventory. Although some of these distracters represent alternative ideas that had been already identified in earlier research, we believe that their systematical presentation through our KMTG inventory can further contribute to research on students' understandings of the kinetic theory of gases.

The KMTG concept inventory covers the following concepts: structure, volume, pressure, intermolecular potential energy, kinetic molecular energy, temperature, average molecular velocity, thermal internal energy, and gas entropy (see Table IV). These concepts were selected with the aim to assess students' understanding of all the diverse kinetic molecular concepts that are currently included in the various introductory physics curricula at different Croatian universities.

In this paper, the above mentioned concepts are used as themes for discussion and organization of specific student difficulties. It should be noted that we do not consider these categories of student difficulties to be perfectly mutually independent. As a matter of fact we, for example, strongly believe that some difficulties with pressure and temperature appear as a result of difficulties with understanding the concept of a particles' average kinetic energy. However, it has been shown that the correlation between students' success on each pair of the given categories is small to medium for all pairs of categories (see Table $\mathrm{V}$ in Appendix B).

Each of the 22 questions consists of an item stem and 5 answering choices, with only one correct answer. 
TABLE IV. Conceptual coverage of the kinetic molecular theory of gases (KMTG) concept inventory.

\begin{tabular}{lc}
\hline \hline Concepts covered by the concept inventory & Tasks \\
\hline $\begin{array}{l}\text { Structure of a gas (arrangement, interactions } \\
\text { and way of movement of molecules) }\end{array}$ & $1,2,5,6$ \\
Volume of a gas & 7 \\
Gas pressure & 14 \\
Intermolecular potential energy & $3,4,8,11$ \\
Average kinetic energy per molecule of a gas & $10,18,22$ \\
$\quad$ and the total kinetic energy of gas molecules & 16,21 \\
Temperature of a gas & 13 \\
Average velocity of gas molecules & $9,12,15,17$ \\
Thermal internal energy of a gas & 19,20 \\
Entropy of a gas & \\
\hline \hline
\end{tabular}

The distractors were created based on results of the already described think alouds.

Questions 1, 2, 5, and 6 were used for exploring students' conceptual understanding of gas structure (arrangement, interaction, and molecular movement). The 1st question was used for investigating students' ability to differentiate between real and ideal gases. With the 2nd question we aimed to assess students' understanding of the Brownian motion of gas molecules. Finally, the 5th and 6th questions were used for exploring students' understanding of molecular velocity distribution.

In the 7th question we have investigated the student ability to predict the change of gas volume with change of temperature.

The 14th question was used for assessing students' qualitative understanding of gas pressure.

When examining thermal internal energy, in the case of ideal gas, we only consider kinetic energy, whereas in the case of real gas, we also take into account intermolecular potential energy. To our knowledge, there are no earlier studies in which this specific conceptual understanding is explored. Therefore, we have focused our attention on researching this specific concept, including it explicitly in four questions $(3,4,8,11)$ and implicitly in another four questions $(9,12,15,17)$. Through these questions we have attempted to investigate students' understanding of dependence of intermolecular potential energy on distance between gas molecules $(3,4)$, gas temperature (8), and number of gas molecules (11).

We have explicitly included the concept of average kinetic molecular energy in three questions $(10,18,22)$. In the 10th question the attention is focused on its dependence on temperature, in the 18th question on its change during heat transfer, and in the 22nd question on its change with the change of vessel volume. Using knowledge of average kinetic molecular energy and total kinetic molecular energy was also required for solving questions on thermal internal energy $(9,12,15,17)$ and gas temperature $(16,21)$.
Questions 16 and 21 were used in an attempt to investigate students' understanding of the link between gas temperature and average kinetic molecular energy. Concretely, in questions 16 and 21 students were required to think about gas temperature during adiabatic gas compression and after heat transfer, respectively.

In question 13 our aim was to investigate students' understanding of the concept of average molecular velocity. Students were expected to consider the dependence of molecular velocity on mass of the gas molecules, for a given temperature.

Results from educational research $[3,4,25,38]$, as well as our teaching experience at different levels of education (from primary school to university) and workshops interactions with school teachers, made us aware of the numerous difficulties that students and even some teachers have with the concept of thermal internal energy [39] of a gas. Therefore we decided to cover this concept in four different questions $(9,12,15,17)$. In the 9 th and 17th questions we asked what will happen with thermal internal energy during the gas cooling process, and during adiabatic gas compression, respectively. In the 12th and 15th questions, respondents were expected to show understanding about the relationship between thermal internal energies and number or mass of molecules, respectively, for a given temperature.

Questions 19 and 20 were aimed to assess students' qualitative understanding of entropy of the thermodynamic state as a measure of the number of microstates that may realize a given macroscopic state.

The ideal gas model can be effectively used for purposes of solving tasks $2,7,10,13,14,16,18$, and 21 . In other words, it can be safely used to solve tasks that include concepts such as molecular movement, gas volume, kinetic molecular energy (its dependence on temperature and its change during heat transfer), molecular velocity, gas pressure, and gas temperature. The remaining tasks require the students to use their understanding of the structure of a gas, intermolecular potential energy, thermal internal energy of a gas, entropy of a gas, and kinetic molecular energy (its change with the change of vessel volume). These tasks cannot be solved by referring to the ideal gas model-the use of the real gas model becomes unavoidable.

Finally, we attempted to obtain a measure of the concept inventory's content validity by surveying 18 physics teachers. For each of the 22 concept inventory items the teachers were expected to decide whether or not the item measures knowledge or processes that are important for understanding kinetic molecular theory at the level of a typical introductory physics courses at the university. In $92 \%$ of cases the answer to our question was positive, whereby the level of interrater agreement amounted to $88 \%$. Furthermore, 16 out of 18 teachers stated that, in general, the concept inventory represents a valid measure of 
students' understanding of the kinetic molecular theory of gases. With the purpose of estimating the teacher buy-in measure for our instrument, we asked them to assign a mark from 5 (I do not like it at all) to 10 (I like it very much) to each of the 22 items. An average mark of 9 has been reported which speaks of a relatively high teacher buy-in.

Preliminary statistical analyses showed that the itemtotal correlation measure was negative for items 1 , 4, and 11 , which means that these items obviously have to be largely modified or dropped out in the next stages of concept inventory development. Cronbach's alpha for the scale consisting of the remaining items amounts to 0.6. This value indicates a relatively low, but still acceptable realiability $[42,43]$. It should be noted that even after removal of items 1,4 , and 11 the average item discrimination index still remains low-it amounts to 0.2 (range 0.0-0.4), whereby in relevant literature indices above 0.3 are recommended [44]. Such low discrimination indices can be primarily explained by the very low average item difficulty index for our sample $\left(D I_{\mathrm{av}}=0.3\right.$, range $\left.0.1-0.5\right)$, which was clearly below the optimal value recommended by Cohen and Swerdlik [45]. These relatively poor psychometric characteristics represent an obvious limitation of the current version of the instrument. However, according to Adams and Wiemann (as cited in Ref. [43]) items with low discrimination indices can provide us with useful information about course effectiveness. Nevertheless, our assessment instrument identifies alternative ideas (and the reasoning behind them) that teaching has not overcome, despite spending many years studying physics in some cases. It also shows that such misconceptions are common to students of different degrees. This would be an essential step to design teaching proposals that overcome those difficulties.

Finally, we have decided that it would be potentially useful to slightly change the wording of certain questions from our inventory. Hence, in the future versions of the concept inventory we plan to rephrase the expression in option B of question 1: replacing "interaction is smaller" with "interaction is weaker," add to question 2 an additional note that the container is filled with gas, and add to the end of the correct answer A in question 5 "and downwards" (i.e., specifying that the peak is moving to the left as well as downwards).

\section{RESULTS AND DISCUSSION}

The percentages of students' responses for each of the 22 multiple choice (A-E) questions are presented in Figs 1-9. To facilitate discussion, the presentation of survey results is organized along the concepts or themes from Table IV. In each subsection the results for the relevant subgroups of tasks are shown. Thereby, for each task five bar diagrams are used to represent the results of the four student groups (MED, EIT, EDU, and RES, as described in Table III), as well as the results of the total sample (ALL, $N=250$ ). The correct answers are represented by black bars.

We also investigated the extent to which students are successful in using the ideal gas (answers from multiple "ideal gas tasks") and real gas model (answers from multiple "real gas tasks") (see Table VI and Table VII in Appendix B).

\section{A. Gas structure (arrangement, interactions, and way of movement of molecules)}

In question 1, students most often chose the wrong assertion A (37\%), which reflects the misconception that ideal gas particles do not collide with each other because they have a negligible volume. The share of answer A has been found to be surprisingly high in all groups, except in EIT. In the RES group answer A was chosen by $75 \%$ of the students. One could find similar thinking in the time before the 20th century when there were no direct experimental data on the size of molecules. As a matter of fact, supporters of the early kinetic molecular theory assumed that the particles of gas are "infinitely small." Since molecules reduced to a point cannot collide, while the kinetic model assumes gas collisions, in later development of kinetic molecular theory the described model has been corrected in

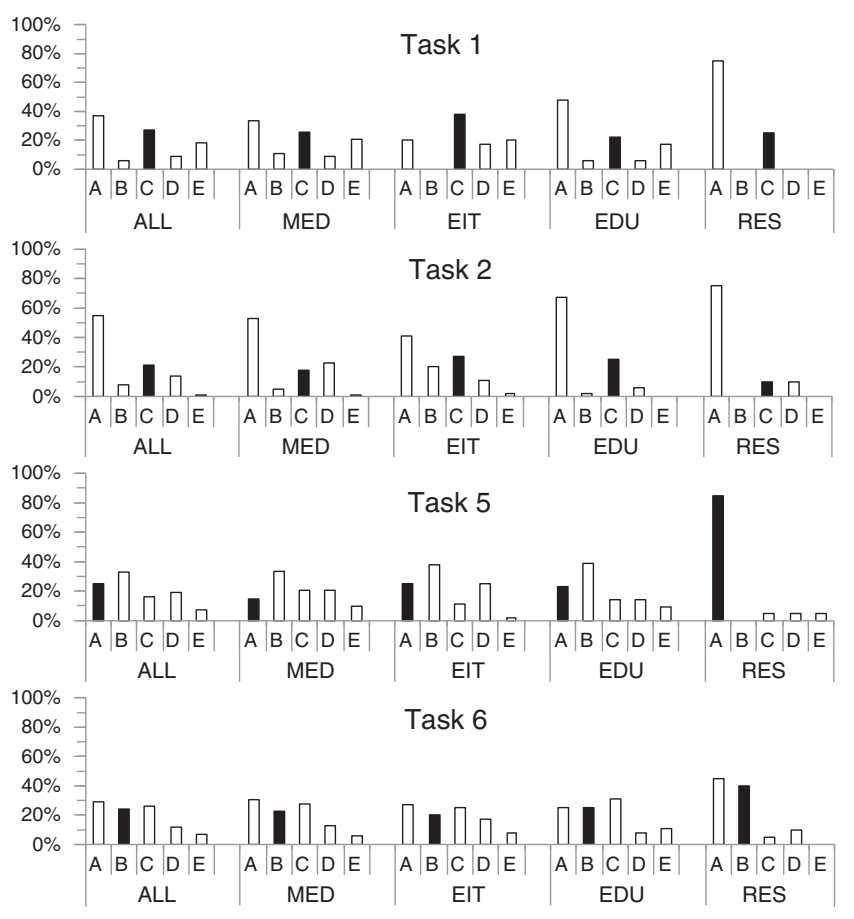

FIG. 1. The students' responses to the concept inventory multiple choice (A-E) questions (Tasks 1, 2, 5, and 6), grouped by the gas structure concept concerned. Results for each question are presented by five bar diagrams, each containing the total sample $(N=250)$ result ALL and the results from all the test groups (MED, EIT, EDU, and RES). The correct answers are presented by the black bars. 
a way that gas molecules were modeled as small balls with finite volume [46].

Nevertheless, many textbooks still quote the obsolete version of the assumption [27], which obviously causes difficulties in conceptual understanding.

Only in the EIT group was the correct answer C the most popular choice $(38 \%)$. Despite dissimilar curricula, for students from the MED and EDU groups similar distributions of responses have been obtained.

In question 2 only $21 \%$ of the total number of students selected the correct path of a gas molecule due to its thermal motion in the vessel, whereby a molecule collides with the walls of the vessel and with other gas molecules (answer C). Between group comparisons show that most correct answers were given by students from the EIT group (27\%).

Although many textbooks explicitly list assumptions about the existence of intermolecular collisions in ideal gases [25], most students (55\%) chose answer A, wrongly believing that gas molecules do not collide with each other, but only with the walls of the container. The answering option A was the most common response in each of the groups, whereby it was most prevalent in the RES group (75\%). Students' reasoning in question 2 seems to be in line with their answers to question 1, where the "negligible particle volume" misconception was identified. In this question, it is also possible that some students reasoned about a separate molecule of gas that moves in the container, which is clearly an unrealistic assumption. However, in the next version of the concept inventory we plan to add an additional note that the container is filled with gas.

If there were no intermolecular collisions then the gas molecules would only collide with walls of the container, as students often mistakenly think. Consequently, the speed of molecules would not be distributed according to the Maxwell distribution function (see questions 5 and 6). From the assumption that all particles of gas enter into the container with equal initial velocities and do not collide with each other (question 6) it would follow that the particle velocity remains unchanged, i.e., the distribution of the velocity would be shown in the graph as a vertical line perpendicular to the horizontal (speed) axis. Of course, such a distribution is not really possible. However, $24 \%$ of respondents came to such a conclusion, as can be seen from the results for question 6 (response B). The between group comparison shows that group RES had the highest percentage (40\%) of correct answers on question 6.

Results from questions 5 and 6 indicate that students very often believe that the distribution of velocities does not depend on intermolecular collisions. In fact, $29 \%$ of students believe that the velocity of particles will be distributed according to Maxwell's distribution function although the particles do not collide with each other (answer A in question 6). Also, the majority of students (33\%) wrongly believe that the top of the curve will just move downward after loss of the fastest particles (response B in question 5).

In fact, because of the existence of intermolecular collisions, loss of the fastest particles will cause a decrease in average velocity and as a result the top of the curve will move towards lower velocities - to the left (response A in question 5). This would lead to a cooling of the gas. The correct answer is prevalent $(85 \%)$ only in group RES. However, high student achievement on question 5 (in the RES group) is not consistent with the relatively poor achievement of RES students on question 6 (40\% of correct answers). These results suggest that in traditional physics instruction students sometimes succeed in acquiring mathematical operational knowledge about the velocity distribution function, but fail to develop the corresponding conceptual understanding.

The distribution of students' answers on questions 5 or 6, are very similar in the groups MED and EDU or in groups MED and EIT, respectively, indicating a general conceptual misunderstanding of Maxwell's distribution. This topic is obviously not receiving sufficient attention during teaching.

\section{B. Gas volume}

Only $32 \%$ of the total number of students were able to correctly identify the approximate arrangement of molecules of cooled gas that does not change the aggregate state (answer A in question 7). On this question, students from the EDU group were more successful than students from the other groups. According to the microscopic model, gas particles interact through very weak short-range interactions and move rectilinearly until they collide with other particles of gas or with the vessel wall. It follows that the volume of gas, regardless of its temperature, is approximately equal to the volume of the container in which the gas is located.

The results of our study show that the majority of students have incorrect predictions about the change of gas volume with temperature. The percentages of wrong answers D (33\%), E (17\%), and C (6\%), indicate the great prevalence of a misconception according to which "particles with less kinetic energy move slower and occupy less space." This wrong idea, which we already had discovered through think alouds, has also been identified by Robertson and Schaffer [27]. In our study, students

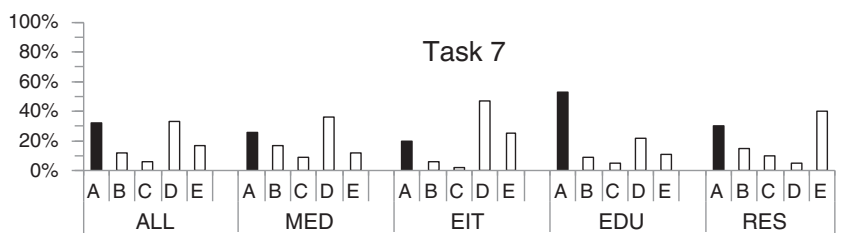

FIG. 2. The students' responses to the task referring to the gas volume concept. For a detailed description see Fig. 1. 
(mostly from MED and EIT groups) often erroneously overestimated the interaction between particles (answer D), which in some cases even resulted in estimating a regular arrangement of particles, such as the one in a solid body, with no volume change (answer B). Answers $\mathrm{C}$ and $\mathrm{E}$ have been chosen by those students who, in addition to intermolecular action, additionally overestimate the particle gravitational interaction with Earth. An alternative explanation of incorrect conclusions on the arrangement of particles of cooled gas would be that these conclusions could also stem from irrelevant observations, such as the observation of contraction of a cooled balloon [27].

In groups MED and EIT, the distributions of students' answers to question 7 were similar.

\section{Gas pressure}

Question 14 has been correctly answered by $33 \%$ of students. These students showed an understanding of the fact that gas pressure does not depend on the mass of molecules (answer A). In all but the EIT group the correct answer A showed to be most prevalent. The highest percentage of correct answers has been observed in group RES (65\%), which can be explained by superior coverage of the corresponding concepts in the curriculum of this group. Question 14 could be simply answered by reasoning about the equation of ideal gas in the form $p V=N k T$. From the equation it is evident that the pressure does not depend on the mass of the molecules, but only on the number of molecules, temperature and volume of the gas.

When it comes to wrong answers, respondents most often (29\% of the total number of students, and even $48 \%$ in EIT group) exhibited a misconception according to which gas pressure depends on the mass of molecules. They probably were misled by the ideal gas equation in the form $p V=n R T$ from which it is evident that pressure $p$ depends on the amount of the substance, i.e., the number of moles of gas $n=m / M$ (E response). Specifically, these students failed to control variables when thinking about this problem, i.e., they focused their attention on the dependence of two variables ( $p$ and $m$ ), ignoring a third $(M)$. Such a pattern of reasoning was already observed in earlier research $[5,13,47]$. In addition to failure to control relevant variables, some students were probably having difficulties with the mere concept of gas. As suggested by Kautz et al. [5], students sometimes attempt to justify their flawed,

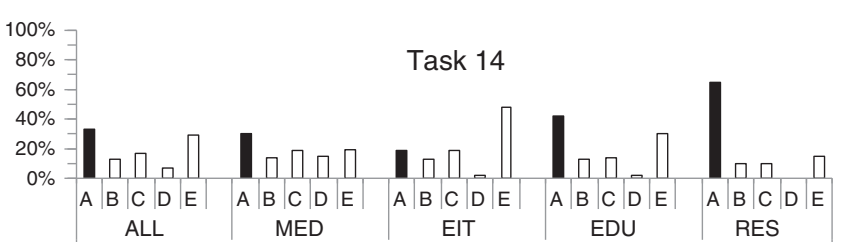

FIG. 3. The students' responses to the task referring to the gas pressure concept. For a detailed description see Fig. 1. qualitative beliefs or preconceptions by misapplying quantitative tools, such as equations.

In question 14, similar distributions of responses were obtained for the EDU and RES groups.

\section{Intermolecular potential energy}

In question 3, only $9 \%$ of the total number of students chose graph A showing the dependence of intermolecular potential energy $U$ on intermolecular distance $r$, for which the average distance between neighboring molecules of gas was properly marked.

The largest number of students chose the wrong answer C (46\%), which was the most prevalent answer in the EDU (58\%) and RES (85\%) groups. Generally, for these two groups of students similar distributions of responses were obtained. For the MED and EIT groups, the distributions of responses were almost identical to each other.

Choosing answering option $\mathrm{C}$ indicates an erroneous transfer of knowledge about intermolecular potential energy in solid matter (which is in the spotlight of traditional instruction) to the context of gases. As a matter of fact, at equilibrium the intermolecular distance in solid matter corresponds to a state for which the system has minimum potential energy (which corresponds to graph C). It is important to note that this statement is valid only at the absolute zero temperature. At higher temperatures, the average distance between neighboring molecules is larger. For gases, the intermolecular average distance is much

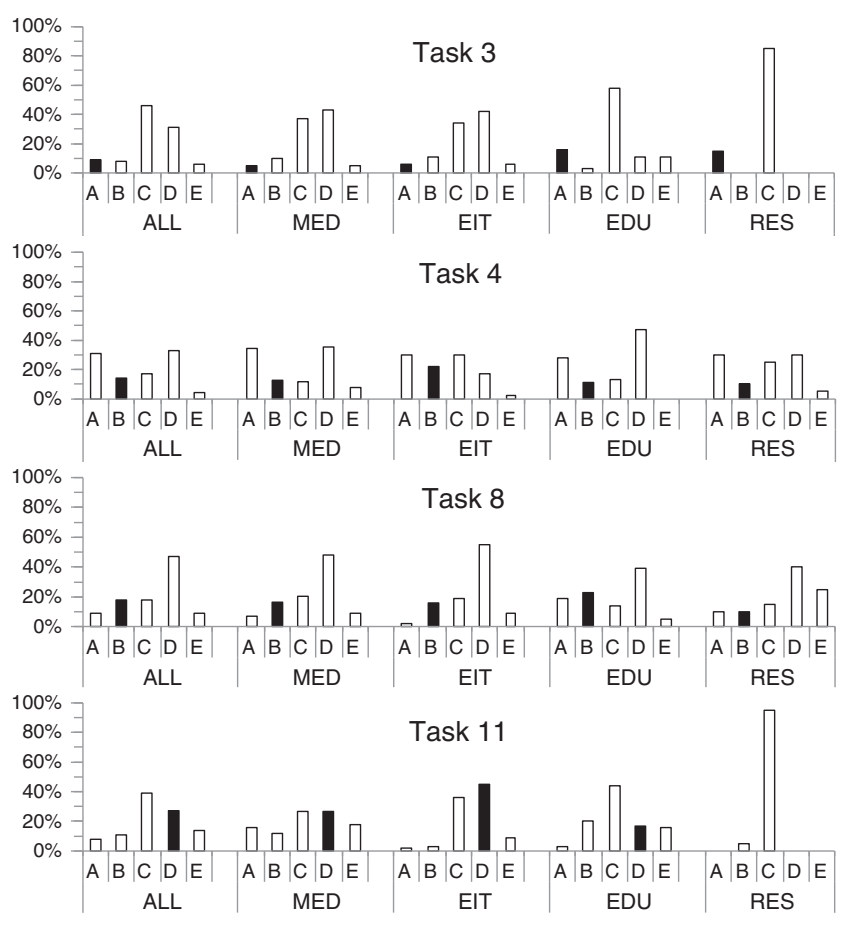

FIG. 4. The students' responses to the tasks grouped by the intermolecular potential energy concept concerned. For a detailed description see Fig. 1. 
larger than for solids. Consequently, the answers B, C, and D can be considered as incorrect.

We suppose that a small percentage of correct answers B to question $4(14 \%)$ results mainly from inaccurate estimates of the average intermolecular distance in the previous question.

The most common incorrect answer was D (33\%). In this answer it is assumed that decreasing intermolecular distance $r$, first leads to a decrease of intermolecular potential energy $U$, after which $U$ increases. Such an answer seems to indicate inconsistency in student reasoning, because in question 3 only $15 \%$ of students chose answer A or E which reflect similar lines of reasoning as answer D in question 4. An alternative explanation would be that students followed similar reasoning as in question 3 , in the sense that in both occasions they attempted to transfer knowledge about solids to the context of gases, i.e., reasoning about the oscillation of molecules of a solid body around equilibrium position, where $U$ decreases, and then increases for particle motion in one direction around the equilibrium position.

For the MED and EDU groups similar distributions of student responses have been obtained.

Questions 8 and 11 were designed for purposes of investigating students' understanding of the relationship between average potential energy of gas molecules and average intermolecular distance, in different contexts.

In question 8 , the correct answer was chosen by only $18 \%$ of the total number of students. They concluded that the average potential energy of gas molecules will not change significantly after cooling of the gas, because the average distance between adjacent gas molecules will not change significantly (answer B). Question 11 was answered correctly by a slightly larger proportion of students (27\%). They correctly concluded that the average potential energy of molecules is greater in that container in which the average distance between neighboring molecules is greater.

Throughout all groups (total 47\%) the most prevalent misconception for question 8 was that after cooling the average potential energy of gas molecules will increase due to a decrease of kinetic energy, and conservation of total energy. Similarly, for question 11, the second most commonly present misconception is distractor E, followed by distractor B. These distractors contain claims about intermolecular potential energy related to "satisfying" conditions of equal thermal internal energies. This result is consistent with findings obtained by Thomas and Schwenz [15] who concluded that some students claim that the energy of a system always remains constant. In our case, it seems that students have mixed up thermal internal energy (which is equal to the sum of kinetic and potential energy of molecules) with total mechanical energy (which is equal to the sum of kinetic and potential energy of the body), identifying thereby intermolecular potential energy with gravitational potential energy. In other words, students misapplied the law of conservation of mechanical energy by ignoring the fact that conservation of energy in thermodynamics is described by the first law of thermodynamics. In thermodynamics, students are expected to reason about the interaction of a physical system with its environment, which then allows them to make conclusions about heat transfer, work, and change of thermal energy. Such reasoning proves to be very difficult for students [4]. We assume that many students tend to avoid such complex reasoning, thereby rather choosing "easier and more familiar or intuitive" arguments like the one contained in question 8's distractor D. An alternative explanation for students' wrong answers in question 8 could also be related to the fact that students often tend to attribute characteristics of macroscopic objects to their particles [48].

For the MED and EIT groups, the distributions of answers to this question proved to be similar to each other.

In question 11 the most prevalent misconception was that decreasing of intermolecular distances leads to an increasing of intermolecular potential energy. Such reasoning is consistent with students' answers to questions 3 and 4 . As a matter of fact, the most commonly chosen distractor in question 3 was $\mathrm{C}$, from which it could be concluded that many students related decreasing of (wrongly identified) average intermolecular distance to increasing of potential energy. Also, there was a relatively large proportion of students who chose A in question 4 , which could be related to the fact that many students have difficulties in distinguishing value and absolute value of a physical variable. Such an interpretation can be corroborated by referring to the think alouds of one interviewed student who stated "Intermolecular potential energy during the transition from gas to liquid increases because bonds are becoming stronger in some ways" (while pointing to part of the curve along which $U$ decreases). In fact, a decrease in the average intermolecular distance in gases results in a decrease of the value of potential energy and with an increase of its absolute value.

\section{E. Average kinetic energy per molecule of a gas and the total kinetic energy of gas molecules}

In question 10, we investigated students' understanding of the concept of average kinetic energy per molecule. The correct answer A was most prevalent in our student sample, chosen by $43 \%$ of students. In the RES group the percentage of correct answers was even 100\%. This result is not very surprising because the RES curriculum mainly promotes a mathematical way of thinking, which is relatively easy to implement within the context of the formula relevant for question 10 (dependence of kinetic energy on temperature). Response A reflects the idea that for two gases at the same temperature, the average kinetic energy per molecule has to be equal, regardless of the different number of molecules in the two gases.

In the total sample, the most frequently chosen distractor was B (40\%), which was particularly prevalent in the 


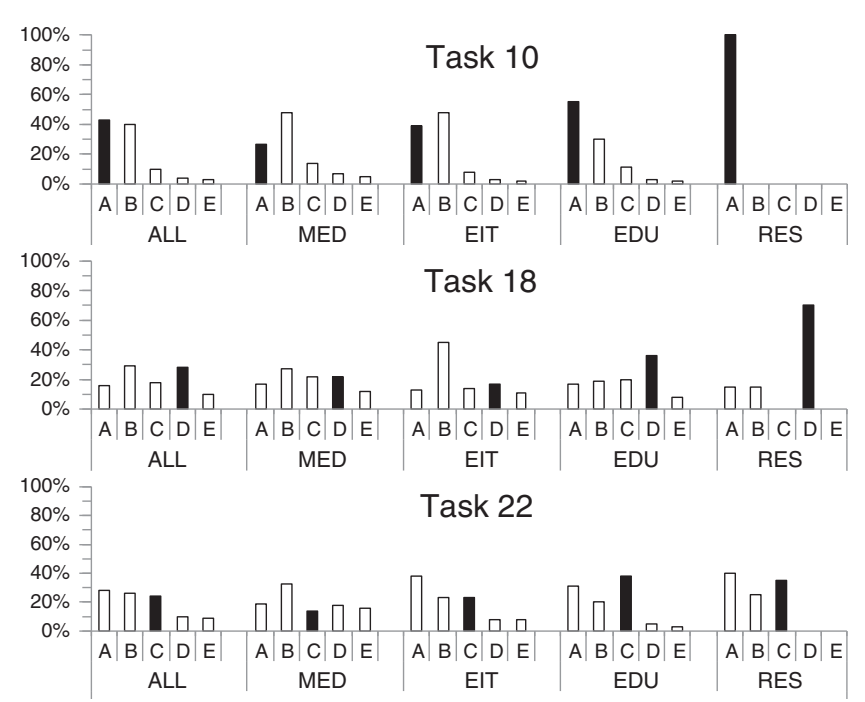

FIG. 5. The students' responses to the tasks grouped by the kinetic energy of gas molecules concept concerned. For a detailed description see Fig. 1.

groups MED (48\%) and EIT (48\%). Distractor B contains the erroneous claim that average kinetic energy per molecule is higher in the container in which particles are closer to each other and more frequently collide. It is interesting to note that many students within the context of question 1 believed that there are no intermolecular collisions in gases, whereas in the context of question 10 they just (erroneously) referred to intermolecular collisions for purposes of explaining the average kinetic energy of molecules. This finding about context specificity of knowledge is consistent with the results of some earlier studies $[3,4,13,25]$. It is known that students' alternative ideas about average kinetic energy of molecules, often include an erroneous microscopic idea that colliding particles produce heat or kinetic energy. It would be correct to reason about the average kinetic energy of molecules of an ideal gas on the basis of energy transfer between gas particles and environment [27], i.e., based on collision of molecules with the moving piston or by referring to heat transfer. However, students often do not reason in that way. For example, they hold the misconception that for a smaller volume ideal gas particles have less space for movements and because of that they collide more frequently. They further erroneously conclude that this results in creating more energy, heat, or friction, which leads to an increase of average kinetic energy per molecule. Moreover, many students often draw an analogy with the behavior of people who, if crowded in a small room, want to get out, look for the exit, and so get warmer [25]. According to Scherr and Robertson [49], the idea of generating thermal internal energy through individual particle collisions is not necessarily an obstacle for learning. It can serve as a productive idea that initiates synchronization of the energy model with a mechanistic understanding of adiabatic compression. Also it can potentially facilitate the development of the correct model of the transformation of kinetic energy into thermal internal energy.

For MED and EIT groups, similar distributions of student responses to question 10 have been obtained.

In question 18 the correct answer (D) was chosen by $28 \%$ of students. Similarly to question 10 , the correct answer was prevalent in the RES (70\%) and EDU (36\%) groups. Respondents who chose answer D, correctly concluded that in the observed situation, after delivering heat to the system, the average kinetic energy per molecule in containers A and B will be approximately the same because of approximately uniform allocation of heat supplied to gas molecules in each container. A relatively large percentage of correct answers in the RES group is not surprising if one compares the breadth or depth of their curriculum to the curriculum of other groups. For this item, a mitigating factor could also be related to the fact that a correct answer could be reached by mere application of the first law of thermodynamics. In fact, by bringing in the same amount of heat, without performing work on the gas, thermal internal energy should increase by the same amount in both containers. Since the contribution of the intermolecular potential energy of the gas is small, this increase of thermal internal energy implies an equal increase of kinetic energy of the gas in both containers. Because of the equal number of particles in both containers, the average kinetic energy will also increase by the same amount.

In question 18, the highest percentage of responses (29\%) has been observed for distractor B, which was especially "popular" amongst students from the EIT (45\%) and MED (27\%) groups. Thereby, the reasoning of these students in the context of question 18 seems to be consistent with their reasoning in question 10. As a matter of fact, in both situations many students claimed that the kinetic energy of the molecules was higher in the container A because of more frequent intermolecular collisions.

Besides the aim of revealing certain misconceptions, question 22 was also supposed to illustrate the fact that the kinetic model of ideal gases is not appropriate for real gases and as such it is not recommended to be used as a conceptual basis for learning thermodynamic concepts [50]. If for purposes of answering question 22 one starts from the model of an ideal gas, they would conclude that the act of opening the valve does not result in energy being added or taken away from gas molecules. Thus, using the model of an ideal gas guides us to an erroneous conclusion that there is no change at all when it comes to thermal internal energy, average kinetic energy of the gas molecules (since intermolecular potential energy is neglected in that model), and temperature of the gas. Such a conclusion is in contradiction with the empirical fact that expansion of gases results in their cooling. On the other hand, the described erroneous conclusion was contained in the most 
prevalent distractor A, which has been chosen by $28 \%$ of students, whereby it proved to be particularly popular in the EIT (38\%) and RES (40\%) groups. This distractor is also characterized by erroneous arguments about thermal insulation of the system.

Students who chose distractor B (26\% of the total sample; $32 \%$ in the MED group) possibly related the less frequent intermolecular collisions to the empirically known decrease in temperature (in accordance with answers to questions 10 and 18).

It should be noted that for correctly answering question 22 , students had to start from the model of real gases. Every fourth student provided a correct answer. They had to recognize that by opening the valve an increase in intermolecular potential energy leads to a decrease in gas temperature as well as to a decrease in kinetic energy of the molecules, while thermal internal energy remains unchanged (answer C). This response was only prevalent in the EDU group (38\%).

\section{F. Gas temperature}

Questions 16 and 21 were aimed at investigating students' understanding of the concept of gas temperature. Generally, the temperature is defined in different ways: "as a variable that can be measured with a thermometer" [2], "as a parameter which will be equal for two objects after they have been in thermal contact long enough" [2], "as the tendency of the object to spontaneously release (or absorb) energy to (or from) the environment" [51], "based on the square root of the average velocity squared of particles" [51], or "based on the statistical definition of entropy, which describes how energy is transferred between two objects which are in thermal interaction" [51]. Therefore, it is not surprising that for students it proves to be difficult to develop an understanding of the concept of temperature. According to Meltzer [3], the proportionality between the average kinetic energy of the particles and the temperature of the ideal gas makes a fundamental connection between the macroscopic and microscopic model of thermodynamics. Combining the microscopic model of the ideal gas with the idea of energy transfer between gas molecules and the environment [27] provides a fruitful framework for explanation of gas temperature changes.

For example, in question 16, as the piston moves downward, a part of the piston's energy is transferred to gas molecules. Therefore, the average kinetic energy per gas molecule increases. Since the average kinetic energy of gas molecules is proportional to gas temperature, the temperature also increases as the piston moves downward (answer D). The correct answer was chosen by $27 \%$ of students, and was prevalent only in the RES group (90\%), which is consistent with the results from questions 10 and 18. This result is not surprising when we know that the RES group's curriculum was much more comprehensive (when it comes to thermodynamics contents) compared to the curriculum of other groups. Similarly as in question 18, a correct answer to question 16 could be obtained by straightforward use of first law of thermodynamics.

In question 21, the correct answer $\mathrm{A}$ is that after receiving heat, the temperature as well as the average kinetic energy will be smaller in the container with larger number of molecules, which is container A. As a matter of fact, before receiving heat, the gases in containers $\mathrm{A}$ and $\mathrm{B}$ were at the same temperatures, and therefore the average kinetic energy of the gas molecules was equal in both containers. In each container, the received heat was evenly distributed among the gas molecules. The total amount of received heat was the same for both containers. However, since in container A there was a larger number of gas particles, the average particle in container $\mathrm{A}$ received a smaller amount of energy than an average particle in container B. Consequently, the average kinetic energy of gas molecules in container A was lower than the average kinetic energy of gas molecules in container B. From the proportionality of the average kinetic energy of gas molecules to temperature, it follows that after receiving energy, the gas temperature in container A was lower than the gas temperature in container B. That answer was chosen by $25 \%$ of students, and was again dominant only in the RES group (90\%), which is consistent with the results from questions 16, 10, and 18.

It should be noted that students (except in the RES group) often gave incorrect answers related to the concept of temperature. For example in question 16 , students most often chose the distractor A (36\%), which was particularly prevalent in the MED (34\%) and EDU groups (47\%). These students erroneously believed that due to thermal insulation of the system, the temperature stays constant as the piston moves downward. Such reasoning seems to be consistent with students' answers to question 22. It is also in line with the findings of Loverude, Kautz, and Heron [4] who concluded that for many students it is difficult to relate thermal insulation to the prevention of

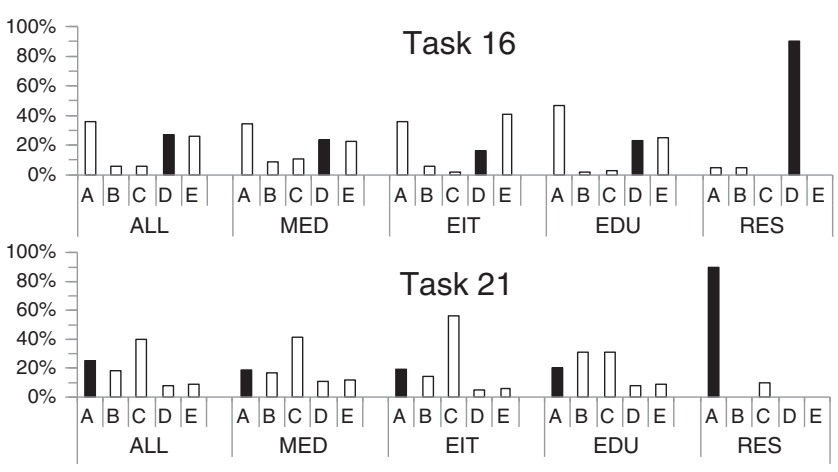

FIG. 6. The students' responses to the tasks grouped by the gas temperature concept concerned. For a detailed description see Fig. 1. 
heat exchange between a system and the environment, as well as to differentiate between thermal internal energy and heat, and to understand the first law of thermodynamics [2-5]. Specifically, Loverude, Kautz, and Heron [4] illustrate students' difficulties applying the first law of thermodynamics for purposes of relating work to change of thermal internal energy or gas temperature in the adiabatic process.

For the MED and EDU groups similar distributions of answers to question 16 have been observed.

In question 21 the highest percentage of students chose the distractor C $(40 \%)$, which was prevalent in the MED (41\%), EIT (56\%), and EDU groups (31\%). This distractor reflects the misconception that a larger number of densely distributed and frequently colliding particles directly implies a higher gas temperature. A similar misconception has been identified in earlier survey research $[25,26]$ and is consistent with the reasoning of many students within the contexts of questions 10, 18, 22, and 16 .

For the MED and EIT groups, similar distributions of answers to question 21 have been observed.

\section{G. Average velocity of gas molecules}

Question 13 has been correctly answered by $28 \%$ of students, whereby the proportion of correct responses was significantly higher in the RES (75\%) and EDU (41\%) subgroups. Students' answers to question 13 showed that they often did not understand that molecules with smaller mass move with greater average velocity in comparison to heavier molecules when they have equal average kinetic energies (answer B). This finding is in accordance with results of the study by Kautz et al. [25].

None of the distractors stood out. For example, students who chose distractor E (18\%) incorrectly believed that lighter molecules are smaller (although this could not be implied from the picture), and for that reason move faster. The misconception that molecules with less mass have to be smaller has already been identified in the study by Kautz et al. [25]. The existence of such a misconception can be an indicator that the student failed to develop a functional knowledge about some basic assumptions of the gas model, according to which the gas molecules can be imagined as small, hard balls that are identical to each other [52]. On the other hand, the described misconception could

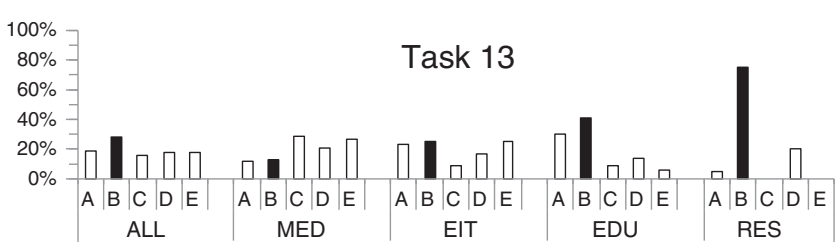

FIG. 7. The students' responses to the task referring to the molecular velocity concept. For a detailed description see Fig. 1. also stem from students' misunderstanding of the relationship between mass and volume [53].

\section{H. Thermal internal energy of a gas}

In question 9 only $16 \%$ of students showed understanding about thermal internal energy of a gas after its cooling. Students have been expected to note that thermal internal energy as well as the average kinetic energy of gas molecules decreases, because the average potential energy of the molecules remained approximately constant (answer A). Answer A was prevalent in the EDU (33\%) and RES (30\%) groups. This is a surprising result because when they were faced with the same physical situation in question 8 many students from the EDU and RES groups claimed that the potential energy increases.

In question 9, the most commonly chosen distractor was E $(32 \%)$, which was a particularly prominent answering option in the EIT group (53\%). Students who chose E seem to believe that the thermal internal energy of the gas decreases due to the decrease of "heat of gas." Such an answer indicates that many students tend to mix up thermal internal energy with heat, which has already been noticed during the think alouds. This finding of ours is in accordance with results of previous studies, e.g., Refs. [3,4,38]. Furthermore, the fact that many students chose the response in which heat is referred to as a property of a gas is also in line with the results of earlier research, e.g., Refs. [54-56]. In many situations it has been shown

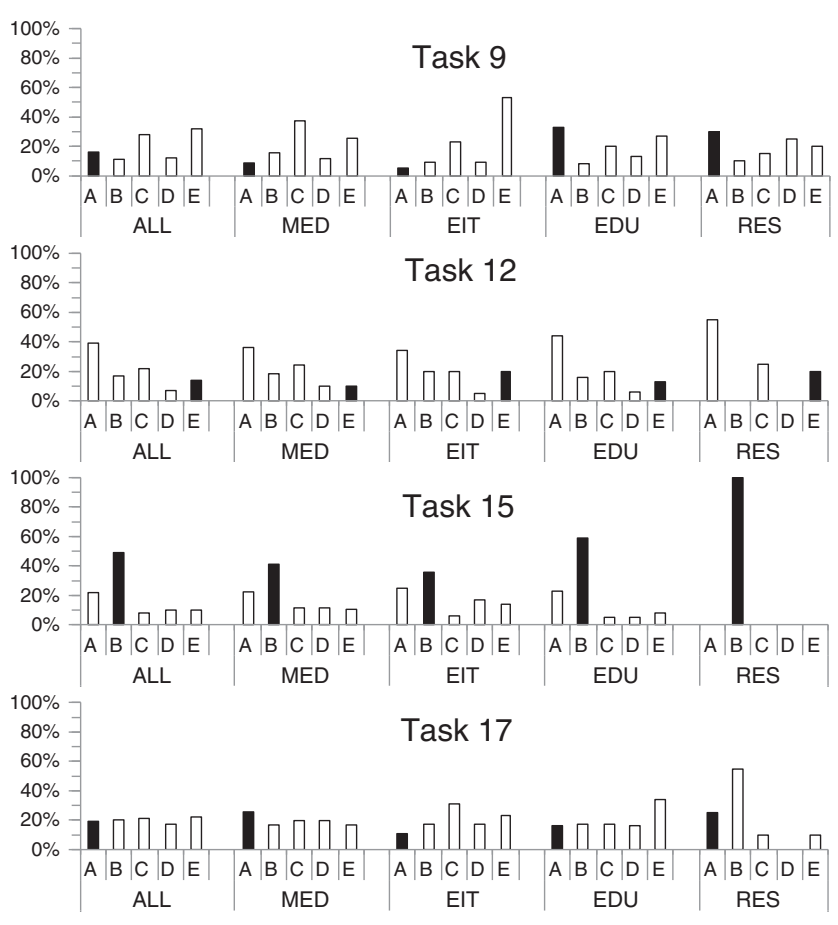

FIG. 8. The students' responses to the tasks grouped by the thermal internal energy of a gas concept concerned. For a detailed description see Fig. 1. 
that students often consider heat to be some kind of a substance or internal property of substance that can enter or leave a physical object, instead of perceiving it as an energy which objects exchange due to temperature differences. These misconceptions reflect ideas that are at the heart of the caloric theory of heat.

While in question 9 we observed the same number of gas molecules at different temperatures, in question 12 we expected the students to compare thermal internal energies of two gases placed in two equal containers containing different numbers of gas molecules, at equal temperatures. The correct answer E was chosen by $14 \%$ of respondents. They correctly concluded that thermal internal energy will be higher in the container with more molecules, mainly due to the contribution of their kinetic energy.

The most commonly chosen distractor was A (39\%), which was prevalent in all groups (EIT, 34\%; MED, 36\%; EDU, 44\%; RES, 55\%). It reflects the misconception that thermal internal energies are equal when temperatures are equal. It seems as if many students tend to mix up thermal internal energy with temperature, which we already noticed during our think alouds, and which is in accordance with results of previous studies (e.g., Ref. [2]).

In question 15, the highest percentage of responses in all groups (EIT, 36\%; MED, 41\%; EDU, 59\%; RES, 100\%) refers to the correct answer B. It contains the claim that for the given situation, thermal internal energy does not depend on the mass of the molecules.

The most common incorrect answer was A (22\%). Students who have chosen that answer mixed up thermal internal energy with mechanical energy. Similar reasoning has been noticed within the context of question 8 , as well as within think alouds. For example, during think alouds one of the students gave the following statement: "In container $B$ there is greater kinetic energy, whereas in container A there is greater potential energy, which means that thermal internal energies for these two containers are equal."

For the MED and EDU groups, similar distributions of responses to the question 15 task have been obtained.

In question 17 students have been expected to conclude that as the piston moves downward, thermal internal energy increases due to an increase in the kinetic energy of molecules of the gas, because their potential energy changes only slightly (answer A). The correct answer has been selected by only $19 \%$ of students.

In the total sample, the most commonly chosen distractor was E (22\%). That answer was most prominent in the EDU group (34\%). Students who chose distractor E erroneously believe that thermal internal energy remains the same as the piston moves downward because temperature and kinetic energy are not changed. Thereby, they exhibit some difficulties understanding of the concept of thermal insulation, and understanding the first law of thermodynamics. Such a conclusion is further corroborated by corresponding think-aloud excerpts, as well as by analyses of student responses on question 16 .

\section{Gas entropy}

Questions 19 and 20 were aimed to assess students' understanding of the concept of entropy. Through qualitative terms [57], the entropy of the macrostate, i.e., of the thermodynamic state, is described as a measure of the number of microstates that may realize a given macroscopic state. Entropy of a macrostate is greater, if the number of its allowed microstates is greater. In other words, entropy depends on the number of ways in which a given macroscopic system can be different at the microscopic level. Consequently, the correct answer to question 19 is that in the larger container the entropy is higher due to a greater number of possible positions of the individual particle. That answer was chosen by $28 \%$ of students. Most successful were the students from the EDU (38\%) and RES groups (65\%).

From the provided qualitative interpretation of entropy, it follows that we cannot talk about entropy at only one instant, but it should be considered over a sufficiently long period of time necessary for establishment of an equilibrium state, as is the case in task 20. In that task, the container is thermally insulated and sealed at room temperature, which means that an equilibrium state is established. Then, the entropy does not change over time (based on the classical Clausius entropy interpretation) as is claimed in the correct answer D in question 20. That answer was chosen by only $14 \%$ of students.

Statements in which entropy is described by referring to concepts of "disorder" or "freedom" are not adequate, as can be shown by examples from statistical mechanics, such as the lattice gas model [57]. However, results of our concept inventory research show that very many students understand entropy just in that way. In question 19, 16\% of students chose answer A, in which higher entropy is related to more irregular arrangement of particles. This distractor was the prevalent response in MED group $(32 \%)$. The larger disorder argument was also mentioned

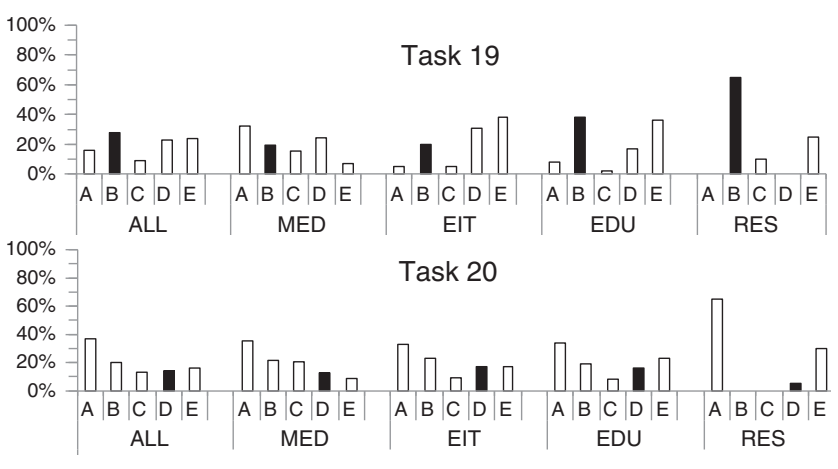

FIG. 9. The students' responses to the tasks grouped by the gas entropy concept concerned. For a detailed description see Fig. 1. 
in distractor D, which was chosen by $23 \%$ of respondents. In distractor $\mathrm{D}$ of question 19 , the bigger disorder of molecules is associated with a larger number of intermolecular collisions. Thus, intermolecular collisions were considered to be very important also in the context of the entropy concept. Similarly, in question 20, the highest percentage of students $(37 \%)$ chose distractor A in which the erroneous conclusion about increasing entropy is explained by greater disorder of the system at the later instant of time (instant 2). That answer was prevalent in all groups (MED, 35\%; EIT, 33\%; EDU, 34\%; RES, 65\%). It is surprising that students from the RES group, who were most successful at solving question 19, now draw incorrect conclusions about entropy based on only one microstate. On the other hand, these results are consistent with Styer's [57] observations. Unlike the students, he perceives configurations (microstates) as constitutive elements of smaller or larger groups (macrostates) that can be associated with less or more entropy. With the help of the lattice gas model it can be demonstrated that configurations that have clusters of particles (or cavities, as is the case with the microstate in instant 1) are typical for a macrostate with greater entropy. Finally, it should be noted that such reasoning is in complete contradiction to human intuition, which could explain the low percentage of correct answers to question 20.

In question 19, the distractor $\mathrm{E}$ was most frequently chosen $(24 \%)$, whereby it was particularly popular in the EIT group (38\%). In distractor E it is stated that entropy is equal in containers $\mathrm{A}$ and $\mathrm{B}$, because they receive the same amount of heat, whereas the systems are insulated and there is no mixing of particles. It seems that students who chose this distractor used the concept of entropy in combination with the second law of thermodynamics, which states that the entropy change of a system is greater than or equal to the sum of entropy changes due to heat transfer [58]. Students who reasoned in the described way possibly did not take into account the fact that before heat had been added to the system entropy was different in the two containers.

For the EIT and EDU groups, similar distributions of student responses to question 20 have been obtained.

\section{J. An overview of students' success on the "ideal gas subscale" and the "real gas subscale"}

If we take the score of $60 \%$ to represent an entry threshold to thinking about ideal gases [59], it can be shown that on average only the students from the physics engineer group reach this threshold (Table VI). Although, for real gases subscale students from all groups were (on average) far from reaching an initial understanding of the concepts measured by this subscale, it could be shown that there is a significant medium sized correlation between students' success on tasks that could be solved with the ideal gas model and their success on tasks that required the use of the real gas model (Table VII). This result could suggest that for students who already have a good understanding of ideal gases it is easier to begin developing an understanding of the real gas model. In other words, our results indicate that mastering of the ideal gas model is often a necessary but not sufficient step to mastering the real gas model. On the other hand it should be noted that sometimes reasoning that was based on the ideal gas model seemed to hinder the students to correctly solve some items that assessed understanding of the real gas model (e.g., intermolecular energy concept). As a matter of fact, although the physics engineer students largely outperformed the students from other groups on "ideal gas tasks," they were ranked last on tasks that were supposed to measure students' understanding of intermolecular potential energy (tasks 4, 8, 11). We can conclude that RES students showed a better understanding of the ideal gas model and it seems that they tried to use it more consistently than students from other groups. However, in certain occasions (e.g., task 8) such reasoning proved to be counterproductive, which eventually resulted in RES students being outperformed by students from other groups.

\section{K. Problems with the ideal gas model: Why the real gas model matters?}

The ideal gas model, as presented in standard textbooks, neglects the intermolecular potential energy which can result in students' difficulties with developing understanding of, e.g., the thermal internal energy concept. In addition, some features of the ideal gas model are presented inconsistently-sometimes the molecules are modeled as balls of small but finite volume, and at other times molecules are described as point masses. Although the ideal-gas law permits us to reason about changes of certain variables, it is important to note that these changes cannot be consistently explained by the ideal gas model. Even correct applications of the ideal gas model can result in wrong conclusions, as we have already emphasized in our discussion of students' responses to task 22. Consequently, we strongly believe that inclusion of the real gas model in introductory physics curricula is of highest importance if we want our students to develop a satisfactory conceptual understanding of gases.

Most of the real gases at low densities behave according to the ideal-gas law, $p V=n R T$. If we start from the hypothesis that the ideal gas law is valid even at very low temperatures $T$ and ambient pressure $p$, we come to the conclusion that the gas volume $V$ tends to zero when absolute temperature $T$ tends to zero. This would be only possible if the gas molecules were a set of noninteracting point masses, which just leads us to the concept of the ideal gas. Many physics textbooks introduce the ideal gas model with the purpose deriving the ideal-gas law and to provide a micromodel for explaining concepts such as temperature 
and pressure. For achieving that aim, it is sufficient to consider elastic collisions of the molecules with the walls of the containing vessel $[61,62]$, whereby the thermodynamic equilibrium, i.e., the Maxwell-Boltzmann velocity distribution, of the gas molecules is presumed. However, the system could never reach thermodynamic equilibrium only through the elastic collisions with the walls. In fact, the state of thermodynamic equilibrium occurs just due to intermolecular collisions. Consequently, to explain the change of the state of the gas, the intermolecular collisions must be introduced. As a matter of fact, in standard textbooks it is typically stated that the ideal gas model permits intermolecular collisions. However, this is inconsistent with the assumption of noninteracting pointlike molecules which have a zero-scattering cross section and collide very seldom or do not collide at all.

We see that, according to typical presentations of the ideal gas model, at the same time the molecules should be large enough to collide among themselves and small enough to satisfy the ideal-gas law. In other words, for any physical model of the gas the ratio of the molecular volume and the volume of the gas $V$ should be a finite number. This is not in line with the main assumption of the ideal gas model which assumes that this ratio is negligible. The ideal-gas model can describe the idealgas law but it cannot satisfactorily explain the change of the state of the gas. The intemolecular collisions can only exist if we assume that ideal gas molecules have a finite volume, as is assumed in the "simplest model of a gas" [46], which is presented as an improvement over the ideal gas model.

Regardless of these conceptual problems, there are no difficulties with mathematical formulation of the ideal-gas law and its application in solving physical problems, including problems with the change of the state of the gas. On the other hand, our results show the existence of numerous problems in the conceptual understanding of the underlying physics. It is thus reasonable to conclude that the ideal-gas law is often understood by the students just as a purely mathematical relationship between physical quantities.

It seems that most of the misconceptions identified in our study are due to an inadequate microscopic picture of the gas. Consequently, we believe that when teaching the kinetic theory of gases a more appropriate approach, compared to introducing ideal gases, is needed. Note that the natural way of introducing the kinetic model of matter in the elementary school is by using the analogy with moving balls, which is already a model of a real gas. As for the university students, the simplest consistent mechanical model of a gas which would satisfy the ergodic hypothesis, thus allowing for change of the state and thermodynamic equilibrium, should be a model which includes a noncentral elastic collision between the balls of finite size, such as dispersing billiards [63].
In our opinion considering the model of ideal gas could be misleading. We believe that it would be more appropriate to introduce the model of a real gas from the mere beginning, followed by considering its simplification which could be described by the phrase "the ideal-gas-law system." An ideal-gas-law system should be considered as a low-density limit of the real gas. In this limit the molecular interactions can be neglected and the total energy can be approximated by the sum of the energies of the individual molecules. But it is important to stress that interactions cannot be completely neglected since thermal equilibrium cannot be established without allowing for intermolecular collisions.

\section{CONCLUSION}

The motivation for our research arose from the fact that informal conversations with students and physics teachers led us to recognize that these populations experience serious difficulties when they attempt to develop understanding of the concept of thermal internal energy in real (everyday) physical contexts. Concretely, many students and teachers applied the model of ideal gases even in such real contexts for which the ideal gas assumptions clearly did not hold, whereby they neglected the intermolecular potential energy contribution to thermal internal energy. Consequently, our informal conversations indicated that many students and physics teachers lack functional mental models of matter that would allow them to comprehend the everyday phenomena in a scientifically appropriate manner. Therefore, we decided to investigate in more detail the student's understanding of the model of real gases, i.e., to investigate not only their understanding of the ideal gas model, but also beyond that model. To that end we created a pool of test items that were aimed to be used for exploring students' understanding of concepts such as the structure of gas, the volume of gas, gas pressure, gas temperature, the average velocity of gas molecules, entropy, thermal internal energy, kinetic energy, and intermolecular potential energy. The open-ended version of the concept inventory has been administered to a sample of university students whose think alouds helped us to design distractors for the closed-ended version of the concept inventory.

Finally, the created concept inventory has been used for surveying 250 students (from different Croatian universities), all of whom had earlier attended thermodynamics classes.

The results of this study support findings from earlier research on student misconceptions. Concretely, the following misconceptions have also been revealed in our study: (i) Gas particles with less kinetic energy move slower and, consequently, occupy a smaller volume [27]. (ii) Molecules of greater mass will generate higher pressure on a given temperature. This misconception could be 
related to students' difficulties with the control of the variables, as well as with their difficulties with the mere concept of a gas $[5,13,47]$. (iii) The intermolecular collisions generate temperature, heat, or kinetic energy of molecules. Therefore, temperature, heat, or kinetic energy of the molecules increases with increase of the frequency of intermolecular collisions, either because of a decrease of the vessel's volume or because of an increase of the number of molecules $[3,4,13,25,26]$. (iv) A thermally insulated gas cannot change its temperature regardless of the work involved [4]. This misconception also reveals misunderstanding of the first law of thermodynamics [2-5]. (v) Thermal internal energy is the same as heat, temperature, or mechanical energy [2-4,25,38]. (vi) Entropy is the result of random arrangement of gas molecules [20,22,57]. Note that the first four above mentioned misconceptions are related to ideal gases, (v) is related to both the ideal and real gases, and (vi) is related to the model of real gases.

Furthermore, we have also identified many misconceptions on the kinetic molecular theory of gases that had not been reported in earlier studies. (i) Because of negligible volume, molecules of a gas do not collide with each other, but only with the walls of the vessel. (ii) The molecular velocity distribution does not depend on intermolecular collisions, but is an inherent characteristic of a gas. (iii) Intermolecular potential energy in a gas is the same as intermolecular potential energy in solid matter, or gravitational energy of the body. Further, intermolecular potential energy in a gas increases as the distance between molecules decreases. (iv) Average kinetic molecular energy of a gas does not change with change of the vessel's volume. Therefore, during expansion the gas will not get colder. This misconception probably stems from students' use of the kinetic molecular theory of ideal gas, which neglects intermolecular potential energy and therefore leads to empirically untenable conclusions.

In addition to a great number of misconceptions and inconsistent answers, we have identified similar distributions of student responses across the many student groups, despite the fact that they came from different teaching environments (i.e., different lecturers, curricula, years of study). This fact can be taken as evidence for a relatively high external validity of our conclusions. It is also surprising that for certain tasks (e.g., see tasks 1, 2, 8, and 11) students who were enrolled in comprehensive thermodynamics courses proved to be less successful compared to students from groups who were enrolled in less comprehensive thermodynamics courses.

We can conclude that the current version of our concept inventory along with the demonstrated methods of its interpretation can be used as a starting point for gaining insight into students' conceptual framework of the kinetic molecular theory of gases. Only based on a good understanding of students' alternative models of gases can we design effective curricula directed at facilitating the development of conceptual models of gases, fluids, and/or solid matter.

Results of this study will be used for further improvement of the KMTG inventory. In addition, findings from this study helped us to develop a tutorial that explicitly addresses some of the difficulties described in this paper. Besides empirical evaluation of the mentioned tutorial by means of the KMTG inventory, in the near future we are also planning to begin developing kinetic molecular theory of liquids and solid matter concept inventories.

\section{ACKNOWLEDGMENTS}

The authors would like to thank all the participants who took part in this study. Special thanks to Professor Ivica Puljak and Professor Nikola Godinović for their help in implementing the concept inventory. I. A. was supported by the Ministry of Science and Education of the Republic of Croatia, under the bilateral CroatiaUSA agreement on the scientific and technological cooperation, Project No. 1/2014. 


\section{APPENDIX A: KINETIC-MOLECULAR THEORY OF GASES CONCEPT INVENTORY}

1. Which of the following statements is true for models of gases? Mark the correct answer.

A) Unlike for a gas, the volumes of the particles for an ideal gas are neglectable which causes the particles not to collide with each other B) In an ideal gas, distant particles interact with each other, but their interaction is smaller than in a gas.

C) Particles in an ideal gas are distributed unevenly like particles in a gas, but unlike them they do not interact with each other when they are far apart from each other.

D) There is no difference between an ideal gas and a gas when it comes to their structure and interactions between particles.

E) An ideal gas is usually represented with small spheres distributed evenly, in contrast to the gas in which the particles are distributed unevenly.

2. A gas molecule is observed in a container represented by the square frame. Which of the following sketches best shows the path of the gas molecule due to its thermal motion? Mark the correct answer.

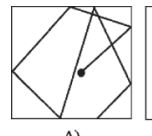

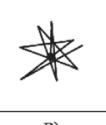

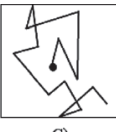

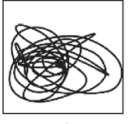

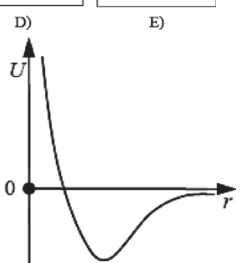

3. The figure shows the dependence of the intermolecular potential energy $U$ on the intermolecular separation $r$. The origin of the coordinate system is set to the position of one of the gas molecules. Which of the following figures correctly shows the average distance $d(\mathrm{O}, \mathrm{P})$ to its first neighbouring gas molecule? Mark the correct answer.

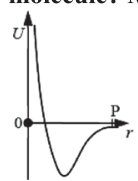

A)

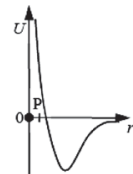

B)

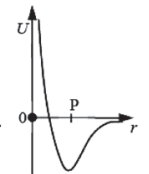

C)

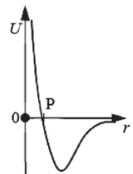

D)

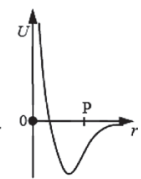

E)
4. How does the intermolecular potential energy change as a consequence of decreasing the average distance between neighbouring gas molecules? Mark the correct answer.

A) Increases, because intermolecular bonds become stronger.

B) Decreases, because the average distance between molecules decreases.

C) Increases, as shown in the diagram from task 3 .

D) First, it decreases to a minimum value and then it increases.

E) Increases, because the particle velocity decreases.

The tasks 5 and 6 refer to the graphical representation of the velocity distribution of gas molecules at room temperature, in a thermally insulated system (see figure).

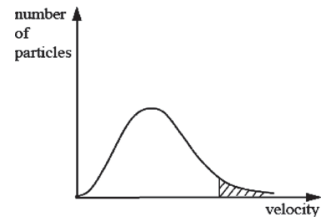

5. How will the velocity distribution look compared to the initial situation after the exclusion of the fastest particles represented by the dashed area in the graph (after some time)? Mark the correct answer.

A) Peak of the curve will move to the left

B) Peak of the curve will move downward, but will not move sideways.

C) Peak of the curve will move to the right.

D) The curve will remain almost the same. Only the dashed area will be missing compared to the initial graph.

E) Peak of the curve will move upward, but it will not move sideways.
6. Suppose that all gas particles from the previous example enter a container with equal initial velocities. How would the velocity distribution of these particles (in the graphical representation) look like after some time provided that the particles do not collide with each other? Mark the correct answer.

A) It will look the same as in the given graph.

B) It will be represented by a length perpendicular to the axis of velocity.

C) It will be represented by a length parallel to the axis of velocity. D) The shape of the curve will be the same as in the given graph, but peak of the curve will be higher and moved to the right compared to the situation from the given graph.

E) The shape of the curve will be the same as in the given graph, but the peak of the curve will be higher compared to the situation from the given graph.

The tasks 7, 8, 9 refer to the following situation. In a sealed vertical container there is gas of temperature $300 \mathrm{~K}$. The given picture shows the instantaneous spatial arrangement of gas molecules (side view of the container's interior). Next, the gas is cooled down to a temperature of $100 \mathrm{~K}$, whithout undergoing phase changes.

7. Which of the pictures best shows the approximate instantaneous spatial arrangement of gas molecules after being cooled down to a temperature of $100 \mathrm{~K}$ ? Mark the correct answer.
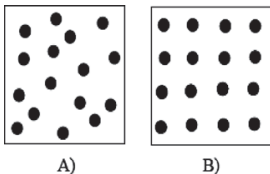

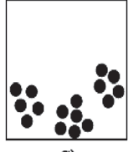

C)

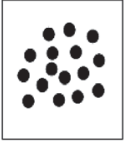

D)

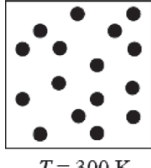

$T=300 \mathrm{~K}$
8. What will happen to the average potential energy of gas molecules after cooling down from $300 \mathrm{~K}$ to $100 \mathrm{~K}$ ? Mark the correct answer.

A) It will not change, because it mostly depends on the number of particles which in this case remains the same.

B) It will not change significantly, because the average distance between gas molecules will not change significantly.

C) It will decrease, because the distance between molecules decreases.

D) It will increase, because the kinetic energy decreases, and the total energy must remain constant.

E) It will increase, because the distance between gas molecules decreases.

9. What will happen to the thermal internal energy of the gas after cooling down from $300 \mathrm{~K}$ to $100 \mathrm{~K}$ ? Mark the correct answer.

A) It will decrease just as it is the case with the average kinetic energy per gas molecule, because the average potential energy of the molecules remains approximately the same.

B) It will decrease, because the number of collisions decreases which leads to a rapid increase of potential energy.

C) It will stay the same, because otherwise the law of conservation of energy would be violated.

D) It will decrease. In addition to the decrease of kinetic energy of the gas molecules, there is also a decrease of potential energy.

E) It will decrease, because gas heat is decreased.

The tasks 10, 11 and 12 refer to the following situation. In two identical sealed containers $A$ and $B$ (shown in the figure) there is a different number of molecules of the same gas. In both containers the temperature of the gas is the same.

FIG. 10. First page of the kinetic molecular theory of gases concept inventory. 


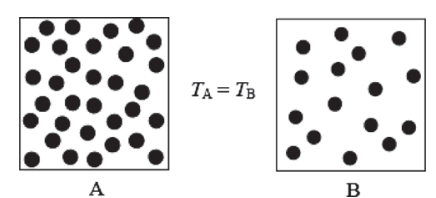

10. Which of the following statements correctly compares the average kinetic energy per molecule for containers $A$ and $B$ ? Mark the correct answer.

A) The average kinetic energy per molecule in containers A and B is equal because of the same temperature.

B) The average kinetic energy per molecule is greater in container A, because the particles are closer together and they more frequently collide.

C) The average kinetic energy per molecule is greater in container $\mathrm{B}$, because of smaller number of particles and less number of collisions.

D) The average kinetic energy per molecule is greater in container $\mathrm{B}$, because the particles will travel a larger distance between two collisions.

E) It is equal in both containers, because in both cases gas molecules move with uniform rectilinear motion.

11. Which of the following statements correctly compares the average potential energy of the molecules in containers $A$ and $B$ ? Mark the correct answer.

A) It is greater in container A due to a greater average kinetic energy per molecule in $\mathrm{A}$.

B) It is greater in container $\mathrm{A}$ because the requirement of equal thermal internal energy must be satisfied.

C) It is greater in container A because of the smaller average intermolecular distance.

D) It is greater in container B because of the larger average intermolecular distance.

E) It is greater in container B because the requirement of equal thermal internal energy must be satisfied.

12. Which of the following statements correctly compares the thermal internal energy of the gas in containers A and B? Mark the correct answer.

A) The thermal internal energy of the gas in container $\mathrm{A}$ is the same as in B because in both cases the temperature of the gas is the same. B) The thermal internal energy of the gas in container A is the same as in B because the total kinetic energy of the molecules is greater in container A, whereas the total potential energy of molecules is greater in container $\mathrm{B}$.

C) The thermal internal energy of the gas is larger in container A because the average potential energy of the molecules is higher in container A.

D) The thermal internal energy of the gas is larger in container B because the kinetic energy is equal as in $\mathrm{A}$, and the potential energy is larger than in A.

E) The thermal internal energy of the gas is larger in container A mainly because of the contribution related to the kinetic energy of a larger number of molecules compared to container B.

The tasks 13, 14 and 15 refer to the following situation. In two equal, sealed containers there is an equal number of gas molecules. The mass of the molecules in container $A$ is larger than the mass of the gas molecules in container B (see picture). The gases are at the same temperature.

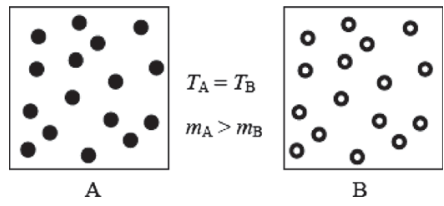

13. Which of the following statements correctly compares the average velocity of gas molecule in containers $A$ and B? Mark the correct answer.

A) The average velocity of the molecule in container $\mathrm{A}$ is the same as in $\mathrm{B}$ because the gases are at the same temperature.

B) The average velocity of the molecule is smaller in container A because the average kinetic energy per molecule is the same for $\mathrm{A}$ and $\mathrm{B}$.

C) The average velocity of the molecule is larger in container A, if the mass of a molecule in A is twice the mass of a molecule in B.

D) The average velocity of the molecule is smaller in container A because of the smaller kinetic energy of molecules in A.

E) The average velocity of the molecule is bigger in container B, because the molecules with lower mass are smaller.

14. Which of the following statements correctly compares the pressure of the gas in containers $A$ and $B$ ? Mark the correct answer.

A) The pressure in container A is the same as in B, because the pressure does not depend on the mass of the molecule.

B) It is higher in container B because the particles with lower mass move faster and collide more often with the container's walls.

C) It is higher in container $\mathrm{A}$, because more massive molecules cause greater changes of the momentum when they collide with the container's walls.

D) It is smaller in container A, since molecules with a greater mass have a greater cross section, and the pressure is defined as force per unit of area.

E) It is higher in container A, because according to the equation-ofstate of ideal gas pressure depends on the amount of substance $n=$ $m / M$.

15. Which of the following statements correctly compares the thermal internal energies of gases in containers A and B? Mark the correct answer.

A) They are equal because the average kinetic energy per molecule is larger in container B, whereas the average potential energy of the molecules is larger in container A.

B) They are equal because in this case the thermal internal energy does not depend on the mass of the molecule.

C) The thermal internal energy is larger in container B, since smaller particles are more firmly bonded and therefore have a greater potential energy.

D) It is larger in container B, because of greater kinetic energy of the molecules, i.e. larger average velocity of the molecules.

E) It is smaller in container B, because the smaller particles are more firmly bonded and therefore have a lower potential energy.

The tasks 16 and 17 refer to the following situation. A thermally insulated cylinder contains a gas. The cylinder is sealed by a piston on its upper side. The piston can slide without friction. Let us consider what happens during the slow movement of the piston to the lower position (see figure).

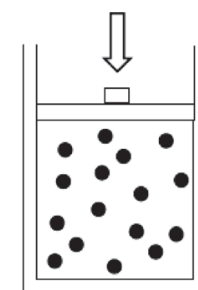

16. What happens to the temperature of the gas as the piston moves downward? Mark the correct answer.

A) It remains the same, because the system is thermally insulated.

B) It remains the same, just as the kinetic energy.

C) It decreases, because it is a compressed gas, just as a deodorant that cools.

D) It increases, because the average kinetic energy per molecule increases.

E) It increases due to an increasing friction between particles that are increasingly colliding and heating up.

FIG. 11. Second page of the kinetic molecular theory of gases concept inventory. 
17. What happens to the thermal internal energy of the gas as the piston moves downward? Mark the correct answer.

A) It increases because the kinetic energy of the molecules increases, and their potential energy only slightly changes.

B) It increases, because the kinetic and potential energy of molecules increase.

C) It increases due to increased heat.

D) It remains the same, because the kinetic energy increases and potential energy decreases.

E) It remains the same, because the temperature, i.e. the kinetic energy does not change.

The tasks 18 and 19 refer to the following situation. The thermally insulated, sealed containers $A$ and $B$ contain the same number of particles of the same gas. The volume of container $B$ is greater than the volume of container $\mathbf{A}$ and the gases are initially at the same temperature. Next, an equal amount of heat is added to the gases (see figure).
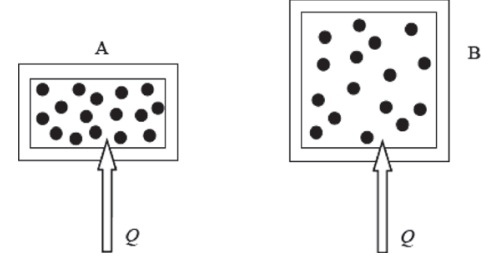

18. Compare the average kinetic energy per molecule in the containers $A$ and $B$, after heat is added to the gases? Mark the correct answer.

A) It will be greater in A, because the molecules are less seperated and they move faster, regardless of heat being added.

B) It will be greater in A, because the molecules in A will collide more frequently.

C) It will be greater in A, due to a smaller volume of the container and greater friction between molecules.

D) It will be approximately equal in both containers, since the added heat is shared approximately equally between the molecules of the gas in each of the contaniers.

E) It will be greater in B, because of the higher temperature in B. The temperature will be higher in the container with the larger volume, i.e. $T \sim V$, which can be inferred from the ideal gas equation.

19. Which of the following statements correctly compares the entropy of gas in containers A and B, after the addition of heat? Mark the correct answer.

A) It will be greater in B due to a less regular arrangement of particles.

B) It will be greater in B, because in a large container there are more possible arrangements of individual particles.

C) It will be greater in B, due to a greater distance between the particles and the greater kinetic energy of particles.

D) It will be greater in A, due to an increasing number of collisions among the particles, causing a bigger disorder in the system.

E) It will be equal in both containers, because equal amounts of heat are added and the systems are insulated so that there is no mixing of particles.

20. The figure shows the arrangement of gas molecules in an thermally insulated, sealed container. The gas is at room temperature and the two figures correspond to two different instants of time ( 1 and 2$)$.
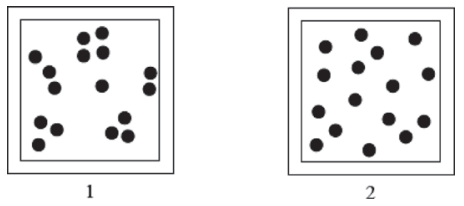

Which of the following statements about entropy is valid for the gas states in instants 1 and 2? Mark the correct answer.

A) Entropy is greater at instant 2, because the system is then less ordered.

B) Entropy is greater at instant 2, because it increases over time.

C) Entropy is greater at instant 1, because at that instant the kinetic energy of the particles is greater which results in more frequent collisions between the particles.

D) At instants 1 and 2 the entropy is equal, because the number of particles does not change.

E) We cannot talk about entropy in this discrete instants of time we can only talk about entropy for sufficient long time intervals.

21. In the thermally insulated, sealed and equal containers $A$ and $B$ there are different numbers of molecules of the same gas. Gases which are initially at the same temperatures, receive equal amounts of heat (see figure).
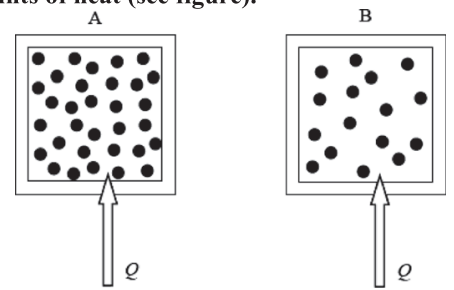

Which of the following statements correctly compares the temperature of gas in containers $A$ and $B$, after the addition of heat? Mark the correct answer.

A) It will be smaller in A (just as the average kinetic energy) because the absorbed heat is approximately evenly shared between a larger number of molecules.

B) It will be equal in both containers (just as the average kinetic energy) due to the receivement of equal amounts of energy.

C) It will be greater in A, due to a larger number of particles which are more densely distributed and which more often collide.

D) It will be greater in B (just as the average kinetic energy) due to the fact that particles now travel larger distances between two subsequent collisions.

E) It is equal, because it is said so in the item stem.

22. A thermally insulated container consists of two parts connected by a narrow tube (see figure). While the valve is closed, the gas is only distributed throughout the left side of the container and in the right part of the container there is a vacuum.

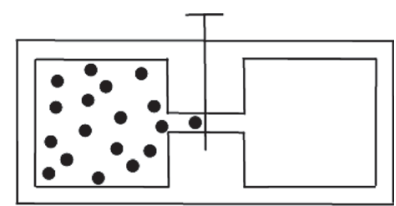

What will happen to the average kinetic energy per gas molecule after the valve is opened? Mark the correct answer.

A) It will remain the same (just as the temperature), because the system is thermally insulated.

B) It will decrease, because the distance between the particles will increase and they will collide less frequently.

C) It will decrease, because the average potential energy will increase, and the thermal internal energy will remain unchanged. D) It will remain the same, just as the thermal internal energy of gas and potential energy of particles of gas, because the gas stays in the same part of the container after the valve is opened.

E) It will increase, because the potential energy of the particles and the thermal internal energy of the gas will increase.

FIG. 12. Third page of the kinetic molecular theory of gases concept inventory. 


\section{APPENDIX B}

TABLE V. Bivariate correlations for students' $(N=250)$ scores on the given task categories.

\begin{tabular}{|c|c|c|c|c|c|c|c|c|c|}
\hline & Structure & Volume & Pressure & $\begin{array}{l}\text { Intermolecular } \\
\text { potential } \\
\text { energy }\end{array}$ & $\begin{array}{l}\text { Kinetic } \\
\text { energy }\end{array}$ & Temperature & $\begin{array}{l}\text { Average } \\
\text { velocity }\end{array}$ & $\begin{array}{l}\text { Thermal } \\
\text { internal } \\
\text { energy }\end{array}$ & Entropy \\
\hline Structure & 1 & 0.01 & 0.00 & 0.08 & $0.16^{\mathrm{a}}$ & $0.15^{\mathrm{a}}$ & 0.06 & 0.11 & 0.04 \\
\hline Volume & 0.01 & 1 & 0.11 & 0.02 & $0.18^{b}$ & 0.04 & 0.10 & 0.10 & 0.11 \\
\hline Pressure & 0.00 & 0.11 & 1 & -0.01 & $0.18^{b}$ & 0.11 & 0.06 & $0.15^{\mathrm{a}}$ & $0.16^{\mathrm{a}}$ \\
\hline $\begin{array}{l}\text { Intermolecular } \\
\text { potential energy }\end{array}$ & 0.08 & 0.02 & -0.01 & 1 & -0.00 & 0.01 & -0.02 & $0.15^{\mathrm{a}}$ & -0.00 \\
\hline Kinetic energy & $0.16^{\mathrm{a}}$ & $0.18^{\mathrm{b}}$ & $0.18^{\mathrm{b}}$ & -0.00 & 1 & $0.29^{\mathrm{b}}$ & $0.17^{\mathrm{b}}$ & $0.13^{\mathrm{a}}$ & $0.23^{\mathrm{b}}$ \\
\hline Temperature & $0.15^{\mathrm{a}}$ & 0.04 & 0.11 & 0.01 & $0.29^{b}$ & 1 & $0.20^{\mathrm{b}}$ & $0.33^{\mathrm{b}}$ & $0.22^{\mathrm{b}}$ \\
\hline Average velocity & 0.06 & 0.10 & 0.06 & -0.02 & $0.17^{\mathrm{b}}$ & $0.20^{\mathrm{b}}$ & 1 & $0.21^{\mathrm{b}}$ & 0.12 \\
\hline $\begin{array}{l}\text { Thermal internal } \\
\text { energy }\end{array}$ & 0.11 & 0.10 & $0.15^{\mathrm{a}}$ & $0.15^{\mathrm{a}}$ & $0.13^{\mathrm{a}}$ & $0.33^{\mathrm{b}}$ & $0.21^{\mathrm{b}}$ & 1 & $0.17^{\mathrm{b}}$ \\
\hline Entropy & 0.04 & 0.11 & $0.16^{\mathrm{a}}$ & -0.00 & $0.23^{\mathrm{b}}$ & $0.22^{\mathrm{b}}$ & 0.12 & $0.17^{\mathrm{b}}$ & 1 \\
\hline
\end{tabular}

${ }^{\mathrm{a}}$ Correlation is significant at the 0.05 level (two-tailed).

${ }^{\mathrm{b}}$ Correlation is significant at the 0.01 level (two-tailed).

TABLE VI. An analysis of between-group differences on the two (ideal gas vs real gas) subgroups of items-minimum, maximum, mean, and standard deviation of the proportion of correct answers are shown.

\begin{tabular}{|c|c|c|c|c|c|c|}
\hline GROUP & & $\mathrm{N}$ & Minimum & Maximum & Mean & Std. Deviation \\
\hline \multirow[t]{2}{*}{ MED } & Real gas & 102 & 0.00 & 0.50 & 0.18 & 0.11 \\
\hline & Ideal gas & 102 & 0.00 & 0.63 & 0.22 & 0.16 \\
\hline \multirow[t]{2}{*}{ EIT } & Real gas & 64 & 0.00 & 0.57 & 0.22 & 0.11 \\
\hline & Ideal gas & 64 & 0.00 & 0.63 & 0.23 & 0.16 \\
\hline \multirow[t]{2}{*}{ EDU } & Real gas & 64 & 0.07 & 0.64 & 0.25 & 0.13 \\
\hline & Ideal gas & 64 & 0.00 & 1.00 & 0.37 & 0.22 \\
\hline \multirow[t]{2}{*}{ RES } & Real gas & 20 & 0.14 & 0.57 & 0.35 & 0.11 \\
\hline & Ideal gas & 20 & 0.38 & 0.88 & 0.66 & 0.14 \\
\hline
\end{tabular}

TABLE VII. Investigating the correlation between students' $(N=250)$ success on the ideal gas and real gas subgroups of items.

\begin{tabular}{llcc}
\hline \hline & & Ideal gas & Real gas \\
\hline Ideal gas & Pearson correlation & 1 & $0.39^{\text {a }}$ \\
& Sig. (two-tailed) & & 0.00 \\
Real gas & Pearson correlation & $0.39^{\mathrm{a}}$ & 1 \\
& Sig. (two-tailed) & 0.00 & \\
\hline \hline
\end{tabular}

${ }^{\mathrm{a}}$ Correlation is significant at the 0.01 level (two-tailed).

[1] R. Leinonen, M. A. Asikainen, and P. E. Hirvonen, Overcoming students' misconceptions concerning thermal physics with the aid of hints and peer interaction during a lecture course, Phys. Rev. ST Phys. Educ. Res. 9, 020112 (2013).

[2] R. Leinonen, E. Rasanen, M. Asikainen, and P. E. Hirvonen, Students pre-knowledge as a guideline in the teaching of introductory thermal physics at university, Eur. J. Phys. 30, 593 (2009).

[3] D. E. Meltzer, Investigation of students' reasoning regarding heat, work, and the first law of thermodynamics in an introductory calculus-based general physics course, Am. J. Phys. 72, 1432 (2004). 
[4] M. E. Loverude, C. H. Kautz, and P. R. L. Heron, Student understanding of the first law of thermodynamics: Relating work to the adiabatic compression of an ideal gas, Am. J. Phys. 70, 137 (2002).

[5] C. H. Kautz, P. R. L. Heron, M. E. Loverude, and L. C. McDermott, Student understanding of the ideal gas law, part 1: A macroscopic perspective, Am. J. Phys. 73, 1055 (2005).

[6] A. G. Harrison, D. J. Grayson, and D. F. Treagust, Investigating a grade 11 student's evolving conceptions of heat and temperature, J. Res. Sci. Teach. 36, 55 (1999).

[7] P. G. Jasien and G. E. Oberem, Understanding of elementary concepts in heat and temperature among college students and K-12 teachers, J. Chem. Educ. 79, 889 (2002).

[8] A. Brook, H. Briggs, B. Bell, and R. Driver, Secondary Students' Ideas about Heat: Workshop Pack, Children's Learning in Science Project (University of Leeds, Centre for Studies in Sci. Math. Educ., Leeds, UK, 1985).

[9] J. Barbera and C. E. Wieman, Effect of a dynamic learning tutorial on undergraduate students' understanding of heat and the first law of thermodynamics, Chem. Educator 14, 45 (2009).

[10] H. Beall, Probing student misconceptions in thermodynamics with in-class writing, J. Chem. Educ. 71, 1056 (1994).

[11] S. Kesidou and R. Duit, Students' conceptions of the second law of thermodynamics - an interpretive study, J. Res. Sci. Teach. 30, 85 (1993).

[12] M. Sozbilir, A review of selected literature on students' misconceptions of heat and temperature, Bogazici University J. Educ. 20, 25 (2003).

[13] S. Rozier and L. Viennot, Students' reasonings in thermodynamics, Int. J. Sci. Educ. 13, 159 (1991).

[14] R. E. Scherr, H. G. Close, E. W. Close, V. J. Flood, S. B. McKagan, A. D. Robertson, L. Seeley, M. C. Wittmann, and S. Vokos, Negotiating energy dynamics through embodied action in a materially structured environment, Phys. Rev. ST Phys. Educ. Res. 9, 020105 (2013).

[15] P. L. Thomas and R. W. Schwenz, College physical chemistry students' conceptions of equilibrium and fundamental thermodynamics, J. Res. Sci. Teach. 35, 1151 (1998).

[16] M. I. Cotignola, C. Bordogna, G. Punte, and O. M. Cappannini, Difficulties in learning thermodynamic concepts: are they linked to the historical development of this field, Sci. Educ. 11, 279 (2002).

[17] R. H. Swendsen, How physicists disagree on the meaning of entropy, Am. J. Phys. 79, 342 (2011).

[18] M. Tribus and E. C. McIrvine, Energy and information (thermodynamics and information theory), Sci. Am. 225, 179 (1971).

[19] T. I. Smith, W. M. Christensen, D. B. Mountcastle, and J. R. Thompson, Identifying student difficulties with entropy, heat engines, and the carnot cycle, Phys. Rev. ST Phys. Educ. Res. 11, 020116 (2015).

[20] M. Loverude, Identifying student resources in reasoning about entropy and the approach to thermal equilibrium, Phys. Rev. ST Phys. Educ. Res. 11, 020118 (2015).

[21] W. M. Christensen, D. E. Meltzer, and C. A. Ogilvie, Student ideas regarding entropy and the second law of thermodynamics in an introductory physics course, Am. J. Phys. 77, 907 (2009).
[22] B. D. Geller, B. W. Dreyfus, J. Gouvea, V. Sawtelle, C. Turpen, and E. F. Redish, Entropy and spontaneity in an introductory physics course for life science students, Am. J. Phys. 82, 394 (2014).

[23] M. Wiser and S. Carey, Mental Models (Erlbaum, Hillsdale, NJ, 1983), pp. 267-297.

[24] P. A. Kraus and S. Vokos, The role of language in the teaching of energy: The case of heat energy, https://spu .edu/depts/physics/documents/WSTA_KrausVokos.pdf (2011).

[25] C. H. Kautz, P. R. L. Heron, P. S. Shaffer, and L. C. McDermott, Student understanding of the ideal gas law, part 1l: A microscopic perspective, Am. J. Phys. 73, 1064 (2005).

[26] A. D. Robertson, Ph.D. thesis, University of Washington, 2011, http://eric.ed.gov/?id=ED534720.

[27] A. D. Robertson and P. S. Shaffer, University student and K-12 teacher reasoning about the basic tenets of kineticmolecular theory, part i: Volume of an ideal gas, Am. J. Phys. 81, 303 (2013).

[28] L. C. McDermott and P. S. Shaffer, Tutorials in Introductory Physics (Pearson, Boston, 2010).

[29] M. Prince, M. Vigeant, and K. Nottis, Development of the heat and energy concept inventory: Preliminary results on the prevalence and persistence of engineering students' misconceptions, J. Eng. Educ. 101, 412 (2012).

[30] K. C. Midkiff, T. A. Litzinger, and D. L. Evans, Development of engineering thermodynamics concept inventory instruments, Frontiers in Educ. Conf. 2, F2A (2001).

[31] S. Yeo and M. Zadnik, Introductory thermal concept evaluation: Assessing students' understanding, Phys. Teach. 39, 496 (2001).

[32] R. Thornton and D. Sokoloff, Heat and temperature concept evaluation, http://physics.dickinson.edu/ wp_ web/wp_resources/wp_assessment.html.

[33] A. Jacobi, J. Martin, J. Mitchell, and T. Newell, A concept inventory for heat transfer, Front. Educ. Conf. 1, T3D (2003).

[34] R. A. Streveler, R. L. Miller, A. I. Santiago-Roman, M. A. Nelson, M. R. Geist, and B. M. Olds, Rigorous Methodology for Concept Inventory Development: Using the 'Assessment Triangle' to Develop and Test the Thermal and Transport Science Concept Inventory (TTCI), Int. J. Eng. Educ. 27, 968 (2011).

[35] S. Prelovsek-Peros, B. Mikulicic, B. Milotic, and I. Aviani, Otkrivamo fiziku 7 (Skolska knjiga, Zagreb, 2014), p. 117.

[36] B. Johnson and L. Christensen, Educational Research, Quantitative, Qualitative, and Mixed Approaches (SAGE Publications, Newberry Park, CA, 2012).

[37] C. J. Johnstone, N. A. Bottsford-Miller, and S. J. Thompson, Technical Report 44, https://nceo.umn.edu/ docs/OnlinePubs/Tech44/TechnicalReport44.pdf.

[38] W. H. Kaper and M. J. Goedhart, Forms of energy', an intermediary language on the road to thermodynamics? part 11, Int. J. Sci. Educ. 24, 119 (2002).

[39] Thermal internal energy is a problematic concept that is often mixed up with internal energy [4]. It is defined as the sum of the kinetic energy of particles or as the sum of potential and kinetic energy of particles that interact [40]. In contrast to thermal (internal) energy, the internal energy 
is defined as the sum of all forms of microscopic energy: $E_{\text {int }}=E_{\text {ther }}+E_{\text {chem }}+E_{\text {nuc }}+\cdots$ [2]. In other words, the internal energy of a substance is the sum of the molecular kinetic energy (due to the random motion of the molecules), the molecular potential energy (due to forces that act between the atoms of a molecule and between molecules), and other types of molecular energy [41].

[40] R. Knight, Physics for Scientists and Engineers with Modern Physics (Addison-Wesley, San Francisco, CA, 2004).

[41] J. D. Cutnell and K. W. Johnson, Physics (John Wiley and Sons, Inc., New York, 1998), p. 359.

[42] A. Bowling, Handbook of Health Research Methods: Investigation, Measurement and Analysis (Open University Press, Maidenhead, 2005), pp. 394-428.

[43] S. B. McKagan, K. K. Perkins, and C. E. Wieman, Design and validation of the quantum mechanics conceptual survey, Phys. Rev. ST Phys. Educ. Res. 6, 020121 (2010).

[44] H.-J. Fisseni, Lehrbuch der psychologischen Diagnostik (Hogrefe-Verlag, Gottingen, 1997).

[45] R. J. Cohen and M. Swerdlik, Psychological Assessment: An Introduction to Tests and Measurements (McGraw-Hill Higher Education, Boston, MA, 2009).

[46] D. Halliday, R. Resnick, and J. Walker, Fundamentals of Physics, 7th ed. (Wiley, New York, 2005), pp. 641-642.

[47] R. Leinonen, M. A. Asikainen, and P. E. Hirvonen, University students explaining adiabatic compression of an ideal gas-a new phenomenon in introductory thermal physics, Res. Sci. Educ. 42, 1165 (2012).

[48] D. Ozalp and A. Kahveci, Diagnostic assessment of student misconceptions about the particulate nature of matter from ontological perspective, Chem. Educ. Res. Pract. 16, 619 (2015).

[49] R. E. Scherr and A. D. Robertson, Productivity of "collisions generate heat" for reconciling an energy model with mechanistic reasoning: A case study, Phys. Rev. ST Phys. Educ. Res. 11, 010111 (2015).

[50] M. K. Summers, Teaching heat-An analysis of misconceptions, Sch. Sci. Rev. 64, 670 (1983).

[51] D. Schroeder, An Introduction to Thermal Physics (Addison-Wesley, San Francisco, CA, 1999).
[52] D. Halliday, R. Resnick, and J. Walker, Fundamentals of Physics (John Wiley \& Sons, New Jersey, 2005), 7 ed., pp. 641-642.

[53] M. E. Loverude, C. H. Kautz, and P. R. L. Heron, Helping students develop an understanding of Archimedes' principle, part i: Research on student understanding, Am. J. Phys. 71, 1178 (2003).

[54] R. Driver, A. Squires, P. Rushworth, and V. WoodRobinson, Making Sense of Secondary Science: Research into Children's Ideas (Routledge, London, 1994).

[55] N. Pizzolato, C. Fazio, R. M. Sperandeo Mineo, and D. Persano Adorno, Open-inquiry driven overcoming of epistemological difficulties in engineering undergraduates: A case study in the context of thermal science, Phys. Rev. ST Phys. Educ. Res. 10, 010107 (2014).

[56] G. L. Erickson, Children's viewpoints of heat: A second look, Sci. Educ. 64, 323 (1980).

[57] D. F. Styer, Insight into entropy, Am. J. Phys. 68, 1090 (2000).

[58] M. J. Cochran and P. R. L. Heron, Development and assessment of research-based tutorials on heat engines and the second law of thermodynamics, Am. J. Phys. 74, 734 (2006).

[59] Similarly, Hestenes and Halloun [60] provides a guideline for interpretation of FCI scores which says that a score of $60 \%$ is an entry threshold to Newtonian thinking, and a score of $85 \%$ and above is an indicator of mastery of the Newtonian force concept.

[60] D. Hestenes and I. Halloun, Interpreting the force concept inventory. A response to Huffman and Heller, Phys. Teach. 33, 502 (1995).

[61] H. D. Young and R. A. Freedman, Sears and Zemansky's University Physics with Modern Physics,, 14th ed. (Pearson Education, Upper Saddle River, NJ, 2016).

[62] R. A. Serway, J. S. Faughn, C. Vuille, and C. A. Bennett, College Physics,, 7th ed. (Thomson Brooks/Cole, Belmont, CA, 2006).

[63] Y. G. Sinai, Dynamical systems with elastic reflections. ergodic properties of dispersing billiard, Russ. Math. Surv. 25, 137 (1970). 